\title{
Meson-meson interactions in a nonperturbative chiral approach
}

\author{
J. A. Oller* and E. Oset ${ }^{\dagger}$ \\ Departamento de Física Teórica and IFIC, Centro Mixto Universidad de Valencia-CSIC, 46100 Burjassot (Valencia), Spain \\ J. R. Peláez \\ Stanford Linear Accelerator Center, Stanford University, Stanford, California 94309
}

(Received 9 April 1998; published 16 February 1999)

\begin{abstract}
A nonperturbative method which combines constraints from chiral symmetry breaking and coupled channel unitarity is used to describe the meson-meson interaction up to about $1.2 \mathrm{GeV}$. The approach uses the $\mathcal{O}\left(p^{2}\right)$ and $\mathcal{O}\left(p^{4}\right)$ chiral Lagrangians. The seven free parameters of the $\mathcal{O}\left(p^{4}\right)$ Lagrangian are fitted to the data. The results are in good agreement with a vast amount of experimental analyses. The amplitudes develop poles in the complex plane corresponding to the $f_{0}, a_{0}, \rho, K^{*}, \phi, \sigma$, and $\kappa$ resonances, the latter two being very broad. The total and partial decay widths of the resonances are also well reproduced. Further extensions and applications of this chiral nonperturbative scheme are also discussed. [S0556-2821(99)05003-1]
\end{abstract}

PACS number(s): 12.39.Fe, 11.80.Et, 13.75.Lb, 14.40.-n

\section{INTRODUCTION}

The meson-meson interaction has been the key problem to test chiral perturbation theory $(\chi \mathrm{PT})$, which has proved rather successful at low energies $[1,2]$. The underlying idea is that an expansion in powers of the meson momenta converges at sufficiently low energy, which in practice is $\sqrt{s}$ $\leqslant 500 \mathrm{MeV}$. However, the convergence at higher energies becomes progressively worse. Even more, one of the peculiar features of the meson-meson interaction is the presence of resonances such as the $\sigma, f_{0}$, or $a_{0}$ in the scalar sector and the $\rho, K^{*}$, or $\phi$ in the vector channels. These resonances will show up in the $T$ matrix as poles that cannot be obtained using standard $\chi \mathrm{PT}$. Nevertheless, the constraints imposed by chiral symmetry breaking are rather powerful and not restricted to the region where $\chi \mathrm{PT}$ is meant to converge [3].

Two independent approaches of a nonperturbative character have extended the use of chiral Lagrangians to higher energies and have been rather successful, reproducing important features of the meson-meson interaction including several resonances. Although these two approaches look in principle rather different, they share a common feature which is the imposition of unitarity. One of them [4,5], based upon the inverse amplitude method (IAM), first suggested in [6], makes use of the lowest order, $\mathcal{O}\left(p^{2}\right)$, as well as the next to leading order, $\mathcal{O}\left(p^{4}\right)$, Lagrangians. Elastic unitarity is imposed and thus no mixture of channels is allowed. Then, the coefficients of the $\mathcal{O}\left(p^{4}\right)$ Lagrangian are fitted to the data. The absence of coupled channels has obvious limitations, but in spite of them, the IAM is able to generate dynamically the $\rho, K^{*}$, and $\sigma$ resonances, and to reproduce $\pi \pi$ scattering in the $(I, J)=(0,0),(1,1),(2,0)$ partial waves, as well as in the $(3 / 2,0),(1 / 2,1)$, and $(1 / 2,0)$ channels of $\pi K$ scattering.

\footnotetext{
*Email address: oller@ @ condor.ific.uv.es

†Email address: oset@condor1.ific.uv.es

‡Email address: pelaez@eucmax.sim.ucm.es; on leave of absence from Departamento de Física Teórica, Universidad Complutense, Madrid, Spain.
}

The results are very successful up to $1 \mathrm{GeV}$ in all these channels but the $(0,0)$, where it only yields good results up to $700 \mathrm{MeV}$. The limitations of this single channel approach become evident, for instance, in the $f_{0}(980)$ and $a_{0}(980)$ resonances ( $J=0$ and $I=0$ and 1 , respectively) which do not appear as poles in the $T$ matrix. The method also has a pathological behavior close to the $T$ matrix zeros [7].

The second approach dealt with the $J=0$ sector alone [8]. The input consists of the $\mathcal{O}\left(p^{2}\right)$ Lagrangian, which is used as the source of a potential between mesons. This potential enters in a set of coupled channel Lippmann-Schwinger (LS) equations (actually closer to Bethe-Salpeter equations, since relativistic propagators are used) which leads to the scattering matrix. The method imposes unitarity in coupled channels; hence it yields inelasticities when inelastic channels open up. Amazingly, the approach has only one free parameter, which is a cutoff that regularizes the loop integrals of the LS equation. Such a method proves rather successful since phase shifts and inelasticities are reproduced accurately up to $1200 \mathrm{MeV}$. The $f_{0}(980)$ and $a_{0}(980)$ resonances appear as poles of the $T$ matrix for $I=0$ and 1 , respectively, and their widths and partial decay widths are very well reproduced. In addition, one finds a pole when $I=0$ at $\sqrt{s}$ $\simeq 500 \mathrm{MeV}$ with a width of around $400 \mathrm{MeV}$, corresponding to the $\sigma$ meson, which was also found with similar properties with the IAM [5].

The appearance of the $f_{0}$ and $a_{0}$ is due to the introduction of the $K \bar{K}$ channel in addition to $\pi \pi$ in $I=0$ and $\pi \eta$ in $I$ $=1$. These resonances disappear if the $K \bar{K}$ channel (not considered in $[4,5])$ is omitted, while the $\sigma$ in $I=0$ is almost not affected. This explains why the $f_{0}$ and $a_{0}$ resonances did not show up in the IAM $[4,5]$.

The success of the scheme of Ref. [8] in the scalar sector gives hope that it could be used in other channels. However, one soon realizes that it does not reproduce properly the $J$ $=1$ sector. This looks less surprising when one recalls that the $\mathcal{O}\left(p^{4}\right)$ chiral Lagrangian can be reproduced with the resonance saturation hypothesis [9], that is, assuming that the actual values of the $\mathcal{O}\left(p^{4}\right)$ parameters are basically saturated by resonance exchanges between Goldstone bosons. In this 
TABLE I. Physical states used in the different $I, J$ channels.

\begin{tabular}{cccccc}
\hline \hline & $I=0$ & $I=1 / 2$ & $I=1$ & $I=3 / 2$ & $I=2$ \\
\hline$J=0$ & $\pi \pi$ & $K \pi$ & $\pi \eta$ & & \\
& $K \bar{K}$ & $K \eta$ & $K \bar{K}$ & & \\
& & $K \pi$ & $\pi \pi$ & \\
$J=1$ & $K \bar{K}$ & $K \eta$ & $K \bar{K}$ & \\
\hline \hline
\end{tabular}

way, one establishes a clear relation between the information contained in the $\mathcal{O}\left(p^{4}\right)$ Lagrangian and the resonances in the meson-meson sector, particularly vector meson resonances, where the approach of [9] has its stronghold. Indeed, the absence of the $\rho$ and $K^{*}$ in the approach of [8], which only uses the $\mathcal{O}\left(p^{2}\right)$ Lagrangian, is an indirect confirmation of the link between these resonances and the $\mathcal{O}\left(p^{4}\right)$ Lagrangian.

The approaches of $[4,5]$ and $[8]$ seem complementary and one may wonder whether there is a generalization of these methods, containing both them as limiting cases. An affirmative answer to this question was recently found and such a generalized method was proposed in [10]. The purpose of the present paper is to exploit the idea of [10] and obtain all the predictions of such an approach in the meson-meson sector, such as phase shifts, inelasticities, resonance properties, etc. At the same time we will establish the links between this scheme and $\chi \mathrm{PT}$ at low energies. We also illustrate qualitatively, using a toy model, why the proposed method is so successful when dealing with amplitudes dominated by resonances.

\section{UNITARY AMPLITUDE IN COUPLED CHANNELS}

Let us write the partial wave decomposition of the mesonmeson amplitude with definite isospin $I$ as

$$
T_{I}=\Sigma_{J}(2 J+1) T_{I J} P_{J}(\cos \theta),
$$

where $T_{I J}$ is the partial wave amplitude with isospin $I$ and angular momentum $J$. In each one of these channels there are several meson-meson states coupled to each other. In Table I, we have listed these states for the $J=0,1$ channels, which contain the most relevant meson-meson information below 1 $\mathrm{GeV}$. Note that it is enough to take into account one or two states in each channel since we are neglecting here, on the one hand, multipion states which are only relevant for higher energies and, on the other hand, the $\eta \eta$ that appears for $(I, J)=(0,0)$. The influence of this state is rather small. We have checked it following the scheme of [8] and, although it has some effect, particularly in the inelasticities, we found it small enough to omit it with the consequent simplicity in the general formalism.

Hence, throughout the present work, $T_{I J}$ will be either a $2 \times 2$ symmetric matrix when two states couple or just a number when there is only one state. In what follows we omit the $I, J$ labels and use a matrix formalism, which will be valid for the general case of $n \times n$ matrices corresponding to $n$ coupled states.

The normalization of $T$ is such that

$$
\frac{d \sigma}{d \Omega}=\frac{1}{64 \pi^{2} s} \frac{k_{f}}{k_{i}}\left|T_{i f}\right|^{2},
$$

where $k_{i}$ and $k_{f}$ are, respectively, the c.m. three momenta of the initial and final state and $s$ is the usual Mandelstam variable. We follow the convention for the sign of $T$ such that in an elastic amplitude $\operatorname{Im} T \leqslant 0$.

Unitarity in coupled channels implies

$$
\operatorname{Im} T_{i f}=T_{i n} \sigma_{n n} T_{n f}^{*},
$$

where $\sigma$ is a real diagonal matrix whose elements account for the phase space of the two meson intermediate states $n$ which are physically accessible. With the normalization that we have chosen, $\sigma$ is given by

$$
\sigma_{n n}(s)=-\frac{k_{n}}{8 \pi \sqrt{s}} \theta\left(s-\left(m_{1 n}+m_{2 n}\right)^{2}\right),
$$

where $k_{n}$ is the on-shell c.m. momentum of the meson in the intermediate state $n$ and $m_{1 n}, m_{2 n}$ the masses of the two mesons in the state $n$.

An equivalent way of expressing the unitarity is by means of the $K$ matrix formalism [11] which states in matrix form that

$$
T^{-1}=K^{-1}-i \sigma,
$$

where $K$ is the $K$ matrix, which is real, and from the former equation is trivially given by

$$
K^{-1}=\operatorname{Re} T^{-1} \text {. }
$$

For the sake of completeness we show briefly the equivalence between Eqs. (3) and (5). Isolating $\sigma$ from Eq. (3) we find

$$
\begin{aligned}
\sigma & =T^{-1} \operatorname{Im} T T^{*-1} \\
& =\frac{1}{2 i} T^{-1}\left(T-T^{*}\right) T^{*-1} \\
& =\frac{1}{2 i}\left(T^{-1 *}-T^{-1}\right)=-\operatorname{Im} T^{-1} .
\end{aligned}
$$

Hence, taking into account Eq. (6) one arrives to Eq. (5) or, equivalently,

$$
T=\left[K^{-1}-i \sigma\right]^{-1}=\left[\operatorname{Re} T^{-1}-i \sigma\right]^{-1} .
$$

This is a practical way to write the $T$ matrix fulfilling unitarity and then the dynamics is contained in the $K$ matrix. In the following we will use the information contained in $\chi \mathrm{PT}$ up to $\mathcal{O}\left(p^{4}\right)$ in order to approximate the $K$ matrix or, equivalently, $\operatorname{Re} T^{-1}$. 
The next point is to realize that the $T$ matrix has poles associated with resonances, which implies that the standard perturbative evaluation of $\chi$ PT will necessarily fail close to these poles. As a consequence, one might try to exploit the expansion of $T^{-1}$, which will have zeros at the poles of $T$, and in principle does not present convergence problems. For illustrative purposes, we can use an analogy with the function $\tan x$ when expanded around $x=0$ ( $x$ playing here the role of $p^{2}$ in the chiral expansion). This function has a pole at $x=\pi / 2$. Its inverse, $\cot x$, has a Laurent expansion around $x=0$ and a zero at $x=\pi / 2$. However, inverting the expansion of $\cot x$ around $x=0$ for values of $x$ near $\pi / 2$ provides a faster convergence than expanding directly $\tan x$ around that point. With this idea in mind let us expand $T^{-1}$ in powers of $p^{2}$ as one would do for $T$ using $\chi \mathrm{PT}$ :

$$
\begin{gathered}
T \simeq T_{2}+T_{4}+\cdots \\
T^{-1} \simeq T_{2}^{-1}\left[1+T_{4} T_{2}^{-1} \cdots\right]^{-1} \simeq T_{2}^{-1}\left[1-T_{4} T_{2}^{-1} \cdots\right] .
\end{gathered}
$$

This expression requires the inversion $T_{2}$ which might not be invertible, as it happens, for instance in the $(1,1)$ channel. In order to avoid the use of $T_{2}^{-1}$ we modify Eq. (8) by formally multiplying by $T_{2} T_{2}^{-1}$ on the right and $T_{2}^{-1} T_{2}$ on the left. All the steps are justified using the continuity of the functions involved in the derivation, starting from a matrix close to $T_{2}$, which can be inverted. Thus, Eq. (8) can be rewritten as

$$
T=T_{2}\left[T_{2} \operatorname{Re} T^{-1} T_{2}-i T_{2} \sigma T_{2}\right]^{-1} T_{2} .
$$

Now, using the expansion for $T^{-1}$ of Eq. (9) we find

$$
T_{2} \operatorname{Re} T^{-1} T_{2} \simeq T_{2}-\operatorname{Re} T_{4}+\cdots
$$

and recalling that

$$
\operatorname{Im} T_{4}=T_{2} \sigma T_{2},
$$

we finally obtain, within the $\mathcal{O}\left(p^{4}\right)$ approximation,

$$
T=T_{2}\left[T_{2}-T_{4}\right]^{-1} T_{2} .
$$

From Eqs. (11) and (6) we also obtain the expression for the $K$ matrix,

$$
K=T_{2}\left[T_{2}-\operatorname{Re} T_{4}\right]^{-1} T_{2},
$$

which is very similar to Eq. (13) but using $\operatorname{Re} T_{4}$ instead of $T_{4}$, which appears in the T matrix formula.

Note, as is clear from Eq. (10), that what we are expanding is actually $T_{2} \operatorname{Re} T^{-1} T_{2}$, which in our analogy would be equivalent to $x^{2} \cot x$, which is also convergent around $x$ $=0$.

In another context, the above equation can also be derived using Padé approximants [12]. This equation is a generalization to multiple coupled channels of the IAM of Refs. [4,5]. It makes the method more general and powerful and also allows one to evaluate transition cross sections as well as inelasticities. Our expansion of $K^{-1}$ in Eq. (11) reminds one of the effective range approximation for elastic scattering at low energies of the quantity $K^{-1}=\sigma \cot \delta$, with $\delta$ the phase shift.

The coupled channel result has additional virtues with respect to the single channel IAM. Indeed, in this latter case the expansion of Eq. (9) is meaningless if $\left|T_{2}\right|<\left|T_{4}\right|$ or $T_{2}$ $=0$ [7]. In particular, if $T_{2}$ vanishes, Eq. (13) yields $T$ $=T_{2}^{2} T_{4}^{-1}$, which has a double zero, whereas the correct result would be $T \simeq T_{4}$. This indeed occurs in the $J=0$ partial waves below threshold (Adler zeros). However, within the coupled channel formalism, if a matrix element, say, $\left(T_{2}\right)_{11}$, vanishes, it is sufficient that $\left(T_{2}\right)_{12} \neq 0$, since then Eq. (13) gives $(T)_{11} \simeq\left(T_{4}\right)_{11}$, which is the correct result. In conclusion, while the single channel IAM gives a double zero whenever $T_{2}=0$, the coupled channel method leads to single zeros close to the zeros of $T_{2}$.

The single channel IAM has another related problem, since close to the Adler zero it presents an spurious pole when $T_{2}=T_{4}$. The coupled channel method also avoids this problem, although it runs into a similar one when the determinant of the $T_{2}-T_{4}$ matrix vanishes below threshold. This happens indeed for $J=0, I=0$ around $\sqrt{s} \simeq 120 \mathrm{MeV}$. Excluding the neighborhood of this zero of the determinant, we can still recover from Eq. (13) the usual $\chi$ PT expansion $T$ $\simeq T_{2}+T_{4}+\cdots$ valid for low energies, typically $|\sqrt{s}|<500$ $\mathrm{MeV}$. In any case we concentrate here on results above the two-pion threshold.

It is now important to realize that Eq. (13) requires the complete evaluation of $T_{4}$, which is rather involved when dealing with many channels, as is the case here. Instead, we present a further approximation to Eq. (13) which turns out to be technically much simpler and rather accurate. In order to illustrate the steps leading to our final formula, let us introduce the diagonal $G$ matrix given by the loop integral with two meson propagators,

$$
G_{n n}(s)=i \int \frac{d^{4} q}{(2 \pi)^{4}} \frac{1}{q^{2}-m_{1 n}^{2}+i \epsilon} \frac{1}{(P-q)^{2}-m_{2 n}^{2}+i \epsilon},
$$

where $P$ is the total initial four-momentum of the two meson system. This $G$ matrix has the property

$$
\operatorname{Im} G_{n n}(s)=\sigma_{n n}(s),
$$

as can be easily checked.

The real part of $G(s)$ is divergent and requires a certain regularization. We evaluate it making use of a cutoff regularization with a maximum value $q_{\max }$ for the modulus of the three-momentum in the integral. An analytical expression for $G_{n n}(s)$ is given in Appendix A.

In a first step let us assume that through a suitable choice of the cutoff we can approximate

$$
\operatorname{Re} T_{4} \simeq T_{2} \operatorname{Re} G T_{2}
$$

or equivalently, from Eq. (14),

$$
K=T_{2}\left[T_{2}-T_{2} \operatorname{Re} G T_{2}\right]^{-1} T_{2}=\left[1-T_{2} \operatorname{Re} G\right]^{-1} T_{2} .
$$


In such a case we go back to Eqs. (10) and (11) and immediately write

$$
T=\left[1-T_{2} G\right]^{-1} T_{2},
$$

which is equivalent to

$$
T=T_{2}+T_{2} G T
$$

which is a LS equation for the $T$ matrix, where $T_{2}$ plays the role of the potential. This is actually the approach followed in Ref. [8].

There is a subtle difference between Eq. (20) and the ordinary LS integral equation. Indeed, Eq. (20) is an algebraic equation since $T_{2}$ and $T$ are factorized out of the integrals with their on-shell value. In contrast, in the ordinary LS equations, the $T_{2} G T$ term is actually the integral of Eq. (15), including $T_{2}$ and $T$ inside the integral, since both of them depend on $q$. As a result of the structure of the $\mathcal{O}\left(p^{2}\right)$ Lagrangian, it was shown in [8] that writing $T_{2}(q)$ as $T_{2}^{\text {on shell }}(q)+T_{2}^{\text {off shell }}(q)$, the off-shell part renormalizes couplings and masses and hence it had to be omitted. Therefore $T_{2}$, and $T$ factorized outside the integral with their onshell values. As a consequence, the very same algebraic equation (20) was obtained.

As we have already commented, the approximation of Eq. (17) leads to excellent results in the scalar channels. However, as we mentioned in the Introduction, the generalization to $J \neq 0$ is not possible since basic information contained in the $\mathcal{O}\left(p^{4}\right)$ chiral Lagrangian is missing in Eq. (17). The obvious solution is to add a term to Eq. (17) such that

$$
\operatorname{Re} T_{4} \simeq T_{4}^{P}+T_{2} \operatorname{Re} G T_{2} .
$$

The $K$ matrix in this case follows immediately:

$$
K=T_{2}\left[T_{2}-T_{4}^{P}-T_{2} \operatorname{Re} G T_{2}\right]^{-1} T_{2},
$$

where $T_{4}^{P}$ is the polynomial tree level contribution coming from the $\mathcal{O}\left(p^{4}\right)$ Lagrangian. As already mentioned in the Introduction there is a link established in [9] between $T_{4}^{P}$ and the exchange of meson resonances, particularly the vector ones. It is now clear from Eq. (22) that through the inclusion of $T_{4}^{P}$ one can generate real poles in the $K$ matrix which will correspond to resonances of the unitarized $T$ matrix. The polynomial $T_{4}^{P}$ is given in terms of the $L_{i}$ coefficients of the $\mathcal{O}\left(p^{4}\right)$ Lagrangian. Within our approach, these coefficients will be fitted to data and denoted by $\hat{L}_{i}$ since they do not have to coincide with those used in $\chi \mathrm{PT}$, as we shall see. Actually, the $L_{i}$ coefficients depend on a regularization scale $(\mu)$. In our scheme this scale dependence appears through the cutoff.

In addition, there are also differences between our renormalization scheme and that of standard $\chi \mathrm{PT}$. Indeed, our approach considers the iteration of loop diagrams in the $s$ channel, but neglects loops in the $u$ or $t$ channel. However, the smooth structure of these terms for the physical $s$ channel, since we are far away from the associated singularities, allows them to be approximately reabsorbed when fitting the $\hat{L}_{i}$ coefficients. Concerning tadpoles, they would be exactly reabsorbed in the $\hat{L}_{i}$ in the equal mass case. Therefore, when masses are different, we are omitting terms proportional to differences between the actual masses squared and an average mass squared. Thus all these contributions will make the $\hat{L}_{i}$ differ from the $L_{i}$, although we expect them to be of the same order.

This way of dealing with tadpoles has an additional advantage. Apart from the usual tadpole diagrams that would also appear in standard $\chi$ PT there are some additional tadpole terms. They come from the determinant of the SU(3) metric that should be included in the path integral measure in order to make the generating functional SU(3) covariant [13]. With dimensional regularization such contributions vanish, but that is not the case when using a cutoff regularization [14]. Nevertheless, we have just described how tadpoles are absorbed within our approximation and thus we do not have to calculate them.

With these approximations our calculations have been considerably simplified at the expense of losing some precision at low energies with respect to the full $\mathcal{O}\left(p^{4}\right) \chi \mathrm{PT}$ calculation. As far as we are mostly interested in resonance behavior as well as higher energies this is not very relevant. Nevertheless, if the complete $\mathcal{O}\left(p^{4}\right)$ calculations were available, we could directly use Eq. (13), and have both an accurate low energy description and a good coupled channel unitarity behavior.

Using Eqs. (10) and (11), our final formula for the $T$ matrix is given by

$$
T=T_{2}\left[T_{2}-T_{4}^{P}-T_{2} G T_{2}\right]^{-1} T_{2} .
$$

\section{TOY MODEL}

In order to illustrate how the method works, we take a simple case of one channel and one amplitude around a resonance which we assume to know exactly. That is,

$$
T=\frac{a p^{2}}{q^{2}-M^{2}+i 2 M \Gamma},
$$

where $p^{2}$ is an invariant quantity, of dimension momentum squared, related to the momenta or masses of the pseudoscalar mesons, $q$ the total four-momentum of the meson pair, and $2 M \Gamma=-a p^{2} \operatorname{Im} G$. The above equation satisfies unitarity exactly as can be seen by using Eq. (7).

To $\mathcal{O}\left(k^{2}\right), k \equiv p, q$, we have

$$
T_{2}=-a \frac{p^{2}}{M^{2}},
$$

whereas at $\mathcal{O}\left(k^{4}\right)$ we have

$$
\operatorname{Re} T_{4}=-\frac{a p^{2} q^{2}}{M^{4}} \equiv T_{2} \frac{q^{2}}{M^{2}}
$$

Then, using Eq. (13) we find 


$$
\begin{aligned}
T & =\frac{T_{2}^{2}}{T_{2}-\operatorname{Re} T_{4}-i T_{2} \operatorname{Im} G T_{2}} \\
& =-\frac{a p^{2}}{M^{2}\left(1-\frac{q^{2}}{M^{2}}+i a \frac{p^{2}}{M^{2}} \operatorname{Im} G\right)} \\
& =\frac{a p^{2}}{q^{2}-M^{2}-i a p^{2} \operatorname{Im} G} .
\end{aligned}
$$

So, as we can see, in this particular case the IAM leads to the exact result for the $T$ matrix, Eq. (24). The result is exact here because $T_{2} \operatorname{Re} T^{-1} T_{2}$ is an $\mathcal{O}\left(k^{4}\right)$ function and hence the expansion up to $\mathcal{O}\left(k^{4}\right)$ in Eq. (11) is exact. However, the structure of Eq. (24) is that of a meson propagator of an unstable particle such as the $f_{0} a_{0}, \rho, K^{*}$, etc. resonances. This could justify why the scheme which we propose works even better that one could naively anticipate, at least for resonant channels.

The above argumentation uses the same power counting in momenta as $\chi \mathrm{PT}$, but presumes that the $\mathcal{O}\left(k^{2}\right)$ amplitude comes from the exchange of a resonance. This seems to be in conflict with [9], where it is shown that resonance exchange contribution shows up at higher orders. However, when taking into account the requirements of short distance behavior of QCD, these two points can be reconciled. In fact, this has been shown, in [15], where a classical vector meson dominance expression for the pion form factor is obtained, in the same lines as Eq. (24), starting from chiral Lagrangians and imposing those QCD constraints at short distances and the large $N_{c}$ limit.

In relation to the previous arguments, the link between unitarized $\chi \mathrm{PT}$ and vector meson dominance has also been discussed in [16].

\section{MATRIX ELEMENTS OF $T_{2}$ AND $T_{4}$}

The lowest order chiral Lagrangian is given by

$$
\mathcal{L}_{2}=\frac{f^{2}}{4}\left\langle\partial_{\mu} U^{\dagger} \partial^{\mu} U+M\left(U+U^{\dagger}\right)\right\rangle,
$$

where $f$ is the pion decay coupling and $\langle\cdots\rangle$ stands for the trace of the $3 \times 3$ matrices built out of $U(\Phi)$ and $M$.

$$
U(\Phi)=\exp (i \sqrt{2} \Phi / f)
$$

where $\Phi$ can be expressed in terms of the Goldstone boson fields as

$$
\Phi(x) \equiv\left(\begin{array}{ccc}
\frac{1}{\sqrt{2}} \pi^{0}+\frac{1}{\sqrt{6}} \eta & \pi^{+} & K^{+} \\
\pi^{-} & -\frac{1}{\sqrt{2}} \pi^{0}+\frac{1}{\sqrt{6}} \eta & K^{0} \\
K^{-} & \bar{K}^{0} & -\frac{2}{\sqrt{6}} \eta
\end{array}\right) .
$$

The mass matrix $M$ is given by

$$
M=\left(\begin{array}{ccc}
m_{\pi}^{2} & 0 & 0 \\
0 & m_{\pi}^{2} & 0 \\
0 & 0 & 2 m_{K}^{2}-m_{\pi}^{2}
\end{array}\right)
$$

where we have assumed the isospin limit $m_{u}=m_{d}$.

The $\mathcal{O}\left(p^{4}\right)$ Lagrangian is given by

$$
\begin{aligned}
\mathcal{L}_{4}= & L_{1}\left\langle\partial_{\mu} U^{\dagger} \partial^{\mu} U\right\rangle^{2}+L_{2}\left\langle\partial_{\mu} U^{\dagger} \partial_{\nu} U\right\rangle\left\langle\partial^{\mu} U^{\dagger} \partial^{\nu} U\right\rangle+L_{3}\left\langle\partial_{\mu} U^{\dagger} \partial^{\mu} U \partial_{\nu} U^{\dagger} \partial^{\nu} U\right\rangle+L_{4}\left\langle\partial_{\mu} U^{\dagger} \partial^{\mu} U\right\rangle\left\langle U^{\dagger} M+M^{\dagger} U\right\rangle \\
& +L_{5}\left\langle\partial_{\mu} U^{\dagger} \partial^{\mu} U\left(U^{+} M+M^{+} U\right)\right\rangle+L_{6}\left\langle U^{\dagger} M+M^{+} U\right\rangle^{2}+L_{7}\left\langle U^{\dagger} M-M^{\dagger} U\right\rangle^{2}+L_{8}\left\langle M^{\dagger} U M^{\dagger} U+U^{\dagger} M U^{\dagger} M\right\rangle,
\end{aligned}
$$

where the terms which couple to external sources are omitted [1].

The states with definite isospin, with the phases $\left|\pi^{+}\right\rangle=$ $-|1,1\rangle,\left|K^{-}\right\rangle=-|1 / 2-1 / 2\rangle$ are given by

$$
\begin{gathered}
\mathbf{I}=\mathbf{0}, \\
|K \bar{K}\rangle=-\frac{1}{\sqrt{2}}\left|K^{+}(\vec{q}) K^{-}(-\vec{q})+K^{0}(\vec{q}) \bar{K}^{0}(-\vec{q})\right\rangle, \\
|\pi \pi\rangle=-\frac{1}{\sqrt{6}} \mid \pi^{+}(\vec{q}) \pi^{-}(-\vec{q})+\pi^{-}(\vec{q}) \pi^{+}(-\vec{q}) \\
\left.+\pi^{0}(\vec{q}) \pi^{0}(-\vec{q})\right\rangle,
\end{gathered}
$$

$$
\mathbf{I}=\mathbf{1}, \quad \mathbf{I}_{\mathbf{3}}=\mathbf{0},
$$

$$
\begin{gathered}
|K \bar{K}\rangle=-\frac{1}{\sqrt{2}}\left|K^{+}(\vec{q}) K^{-}(-\vec{q})-K^{0}(\vec{q}) \bar{K}^{0}(-\vec{q})\right\rangle, \\
|\pi \eta\rangle=\left|\pi^{0}(\vec{q}) \eta(-\vec{q})\right\rangle, \\
|\pi \pi\rangle=-\frac{1}{2}\left|\pi^{+}(\vec{q}) \pi^{-}(-\vec{q})-\pi^{-}(\vec{q}) \pi^{+}(-\vec{q})\right\rangle,
\end{gathered}
$$




$$
\begin{gathered}
\mathbf{I}=\mathbf{2}, \quad \mathbf{I}_{\mathbf{3}}=\mathbf{2}, \\
|\pi \pi\rangle=\frac{1}{\sqrt{2}}\left|\pi^{+}(\vec{q}) \pi^{+}(-\vec{q})\right\rangle, \\
\mathbf{I}=\mathbf{1} / \mathbf{2}, \quad \mathbf{I}_{\mathbf{3}}=\mathbf{1} / \mathbf{2}, \\
|K \pi\rangle=-\left|\frac{\sqrt{2}}{3} \pi^{+}(\vec{q}) K^{0}(-\vec{q})+\frac{1}{\sqrt{3}} \pi^{0}(\vec{q}) K^{+}(-\vec{q})\right\rangle, \\
|K \eta\rangle=\left|K^{+}(\vec{q}) \eta(-\vec{q})\right\rangle, \\
I=3 / 2, I_{3}=3 / 2, \\
|K \pi\rangle=-\left|K^{+}(\vec{q}) \pi^{+}(-\vec{q})\right\rangle,
\end{gathered}
$$

We should note that in the states of identical particles we have included an extra $1 / \sqrt{2}$ factor in the normalization. This is done to ensure that the resolution of the identity gives unity [recall that $\Sigma_{q}\left|\pi^{0}(\vec{q}) \pi^{0}(-\vec{q})\right\rangle\left\langle\pi^{0}(\vec{q}) \pi^{0}(-\vec{q})\right|=2$ with the states $\pi^{0}(\vec{q}) \pi^{0}(-\vec{q})$ normalized to unity]. This normalization yields the ordinary unitarity formulas, Eq. (3), which we are using to extract phase shifts and inelasticities. However, we should return to the proper normalization at the end in order to obtain the physical amplitudes.

The amplitudes which we obtain are compiled in Appendix B. The projection over each partial wave $J$ is done by means of

$$
T_{I J}=\frac{1}{2} \int_{-1}^{1} P_{J}(\cos \theta) T_{I}(\theta) d(\cos \theta) .
$$

In the case of two coupled channels, $T_{I J}$ is a $2 \times 2$ matrix whose elements $\left(T_{I J}\right)_{i j}$ are related to $S$ matrix elements through the equations (omitting the $I, J$ labels)

$$
\begin{aligned}
& (T)_{11}=-\frac{8 \pi \sqrt{s}}{2 i p_{1}}\left[(S)_{11}-1\right], \quad(T)_{22}=-\frac{8 \pi \sqrt{s}}{2 i p_{2}}\left[(S)_{22}-1\right], \\
& (T)_{12}=(T)_{21}=-\frac{8 \pi \sqrt{s}}{2 i \sqrt{p_{1} p_{2}}}(S)_{12},
\end{aligned}
$$

with $p_{1}, p_{2}$ the c.m. momenta of the mesons in state 1 or 2 , respectively. The $S$ matrix has the structure [17]

$$
S=\left[\begin{array}{cc}
\eta e^{2 i \delta_{1}} & i\left(1-\eta^{2}\right)^{1 / 2} e^{i\left(\delta_{1}+\delta_{2}\right)} \\
i\left(1-\eta^{2}\right)^{1 / 2} e^{i\left(\delta_{1}+\delta_{2}\right)} & \eta e^{2 i \delta_{2}}
\end{array}\right],
$$

where $\delta_{1}$ and $\delta_{2}$ are the phase shifts for the elastic $1 \rightarrow 1$ and $2 \rightarrow 2$ processes [for instance, $\bar{K} K \rightarrow \bar{K} K$ and $\pi \pi \rightarrow \pi \pi$ in $(I, J)=(0,0)]$ and $\eta$ is the inelasticity.

It is interesting to note that, by means of $(T)_{11}$ and $(T)_{22}$, one can determine $\eta, \delta_{1}$, and $\delta_{2}$, and hence the $(T)_{12}$ $=(T)_{21}$ matrix elements are redundant. We determine them from our coupled equations and verify that the structure of Eq. (34) is satisfied, which is another check of the coupled channel unitary that we have imposed from the beginning.

\section{RESULTS}

We have carried out a fit to the data, which is shown in Figs. 1-7, using as free parameters the $\hat{L}_{i}$ with $i$ $=1,2,3,4,5,7$ and $2 \hat{L}_{6}+\hat{L}_{8}$. The cutoff is fixed to $q_{\max }$ $=1.02 \mathrm{GeV}$. The values which we obtain are shown in Table II. By comparing them with the standard values for the $L_{i}$ coefficients obtained in $\chi P T$ at the scale $\mu=2 q_{\max } / \sqrt{e}$ (see Appendix A 2) we see that they are of the same order.

We show first the results on phase shifts and inelasticities in the different channels and later on we discuss the pole positions, widths, and partial decay widths.

\section{A. Phase shifts and inelasticities}

We will now go in detail through the results in each $(I, J)$ channel.

\section{Channel $(0,0)$}

As we can see in Eq. (34) we have three independent magnitudes $\delta_{1}, \delta_{2}$, and $\eta$. In Figs. 1(a) and 1(c) we show the $\delta_{1}$ and $\delta_{2}$ corresponding to $\pi \pi \rightarrow \pi \pi$ and $K \bar{K} \rightarrow K \bar{K}$ elastic scattering. In Fig.1(b) we plot the phase shift for $K \bar{K} \rightarrow \pi \pi$. This is actually $\delta_{1}+\delta_{2}$, which is therefore redundant information. However, there are data for this process but not for elastic $K \bar{K}$, and that is why we are plotting $\delta_{1}$ $+\delta_{2}$. The agreement with experiment is good, with small discrepancies in the $K \bar{K} \rightarrow \pi \pi$ phase shifts. In Fig. 1(a) we see a bump around $600 \mathrm{MeV}$ which is due to the $\sigma$ resonance, whose associated pole appears around 442-i225 $\mathrm{MeV}$, as we shall see below. The fast raise in the phase shift at $1 \mathrm{GeV}$ is caused by the $f_{0}$ pole around $980-i 14 \mathrm{MeV}$, which translates into an apparent mass of $\simeq 980 \mathrm{MeV}$ and a $30 \mathrm{MeV}$ width. Small discrepancies with the data start show-

TABLE II. Fit parameters $\hat{L}_{i} \times 10^{3}$ and comparison with the $L_{i}^{r} \times 10^{3}$ of $\chi \mathrm{PT}$.

\begin{tabular}{lccccccc}
\hline \hline$q_{\max }=1.02 \mathrm{GeV}$ & $\hat{L}_{1}$ & $\hat{L}_{2}$ & $\hat{L}_{3}$ & $\hat{L}_{4}$ & $\hat{L}_{5}$ & $2 \hat{L}_{6}+\hat{L}_{8}$ & $\hat{L}_{7}$ \\
& 0.5 & 1.0 & -3.2 & -0.6 & 1.7 & 0.8 & 0.2 \\
$\mu=1.2$ & $L_{1}^{r}$ & $L_{2}^{r}$ & $L_{3}$ & $L_{4}^{r}$ & $L_{5}^{r}$ & $2 L_{6}^{r}+L_{8}^{r}$ & $L_{7}$ \\
& 0.1 & 0.9 & -3.5 & 0.7 & 0.4 & 0.0 & -0.4 \\
& \pm 0.3 & \pm 0.3 & \pm 1.1 & \pm 0.5 & \pm 0.5 & \pm 0.3 & \pm 0.2 \\
\hline \hline
\end{tabular}



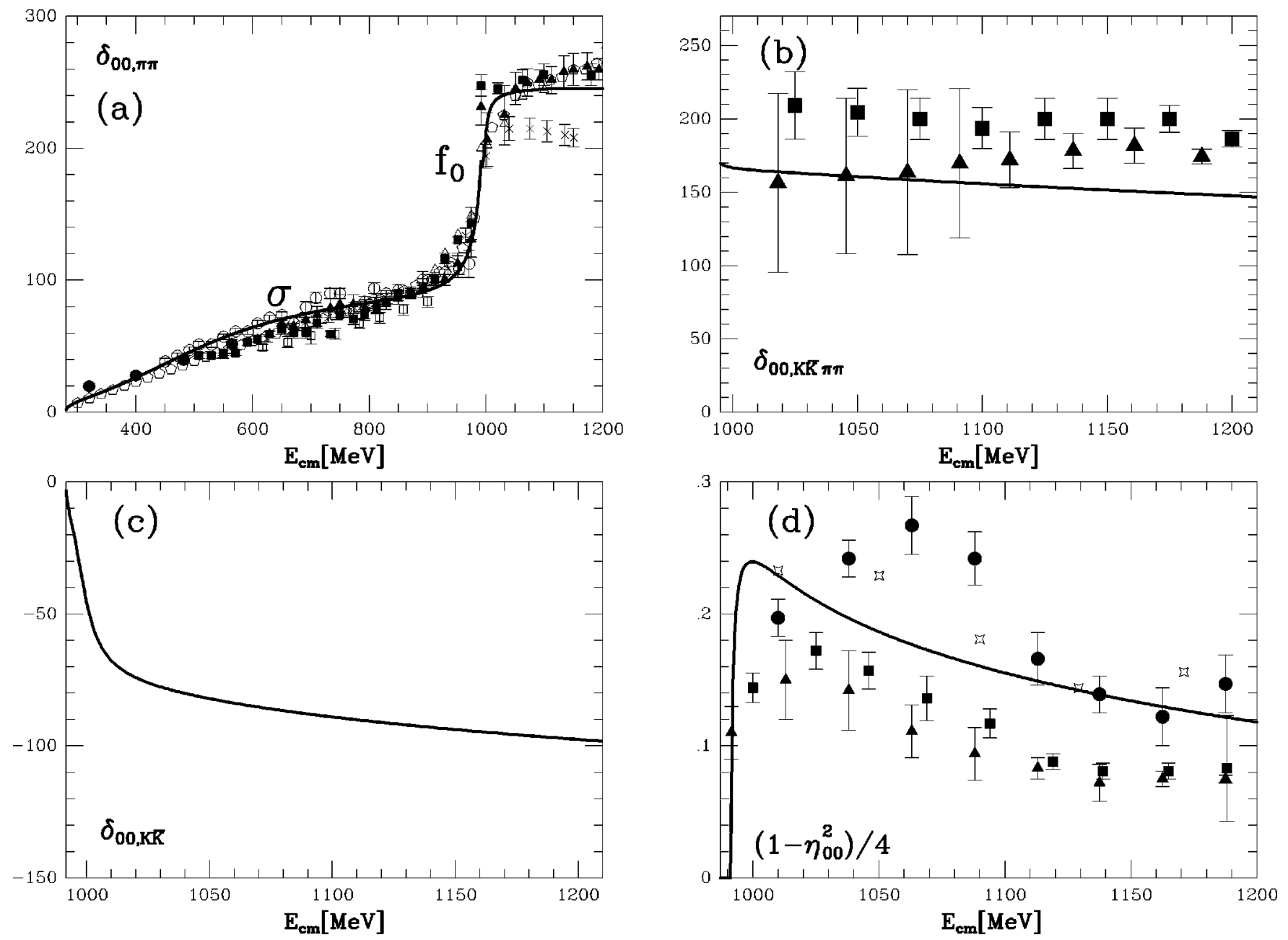

FIG. 1. Results in the $I=J=0$ channel. (a) Phase shifts for $\pi \pi \rightarrow \pi \pi$ as a fraction of the c.m. energy of the meson pair: solid triangle [19], open circle [20], solid square [21], open triangle [22], open square [23] (all these are an analysis of the same experiment [18]), cross [24], solid circle [25], open pentagon [26]. (b) Phase shifts for $K \bar{K} \rightarrow \pi \pi$ : solid square [27], solid triangle [28]. (c) Phase shifts for $K \bar{K}$ $\rightarrow K \bar{K}$. (d) Inelasticity: results and data for $\left(1-\eta^{2}\right) / 4$ : starred square [26], solid square [27], solid triangle [28], solid circle [29].

ing up around $1.2 \mathrm{GeV}$. The omission of the $\eta \eta$ and fourmeson states should limit the validity of the approach at high energies since then these channels start being relevant.

\section{Channel $(1,1)$}

In Fig. 2(a) we display the $\pi \pi \rightarrow \pi \pi$ phase shifts which clearly show the $\rho$ meson. The perfect coincidence of the results with the very precise data indicate that both the position and the width of the $\rho$ are very well described. In Fig. 2(c) we show the phase shifts for $K \bar{K} \rightarrow K \bar{K}$ scattering, for which there are no data. As we can see, they are very small, which implies a weak $K \bar{K}$ interaction. Therefore the $\delta_{1}$ $+\delta_{2}$ phase shift of $K \bar{K} \rightarrow \pi \pi$ is essentially that of $\pi \pi$ $\rightarrow \pi \pi$. The fact that the inelasticity is practically 1 , indicates that there is almost no mixture of $\pi \pi$ and $K \bar{K}$. This feature causes the $\rho$ to behave as a pure $\pi \pi$ elastic resonance. That is why the single channel IAM gave essentially the same results as obtained here [4].

\section{Channel $(2,0)$}

The $I=2 \pi \pi$ scattering contains only one state as shown in Table I. In Fig. 3 we show the resulting phase shifts, whose agreement with experimental data is remarkably good up to $1.2 \mathrm{GeV}$.

\section{Channel $(1,0)$}

In Fig. 4(a) the $\pi \eta \rightarrow \pi \eta$ phase shifts are shown. Those of $K \bar{K} \rightarrow K \bar{K}$ are plotted in Fig. 4(b) and the inelasticities in Fig. 4(c). In the latter, it can be seen that there is an appreciable mixture between $\pi \eta$ and $K \bar{K}$ above $K \bar{K}$ threshold. In Fig. 4(d) we compare a mass distribution for $\pi \eta$ around the region of the $a_{0}$ resonance. The data are obtained from [34] using the $K^{-} p \rightarrow \Sigma^{+}(1385) \pi \eta$ reaction, whose cross section (following [35]) can be written as

$$
\frac{d \sigma}{d m}=C|t|^{2} q
$$



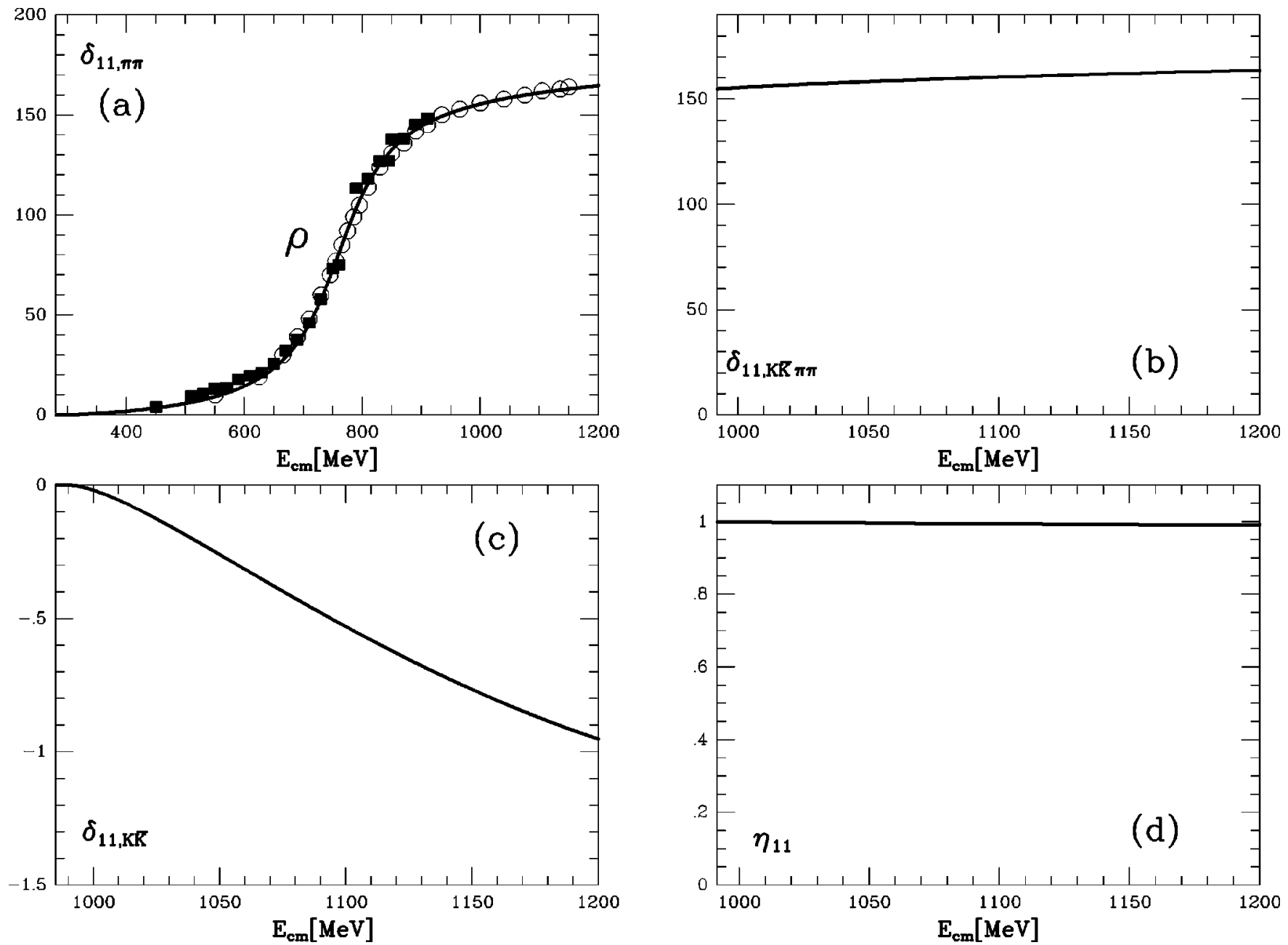

FIG. 2. Results in the $I=J=1$ channel. (a) Phase shifts for $\pi \pi \rightarrow \pi \pi$. Data: open circle [24], solid square [30]. (b), (c) Same as in Fig. 1. (d) Inelasticity.

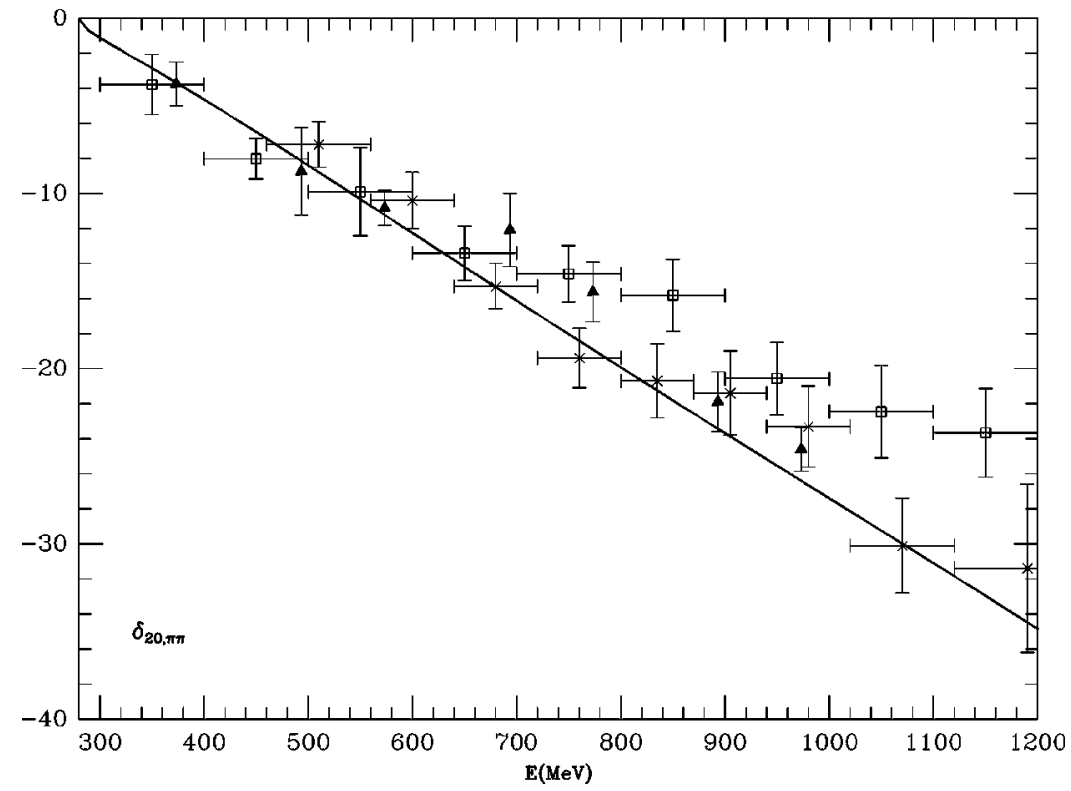

FIG. 3. Phase shifts for $\pi \pi \rightarrow \pi \pi$ in the $I=2, J=0$ channel. Data: cross [31], open square [32], solid triangle [33]. 

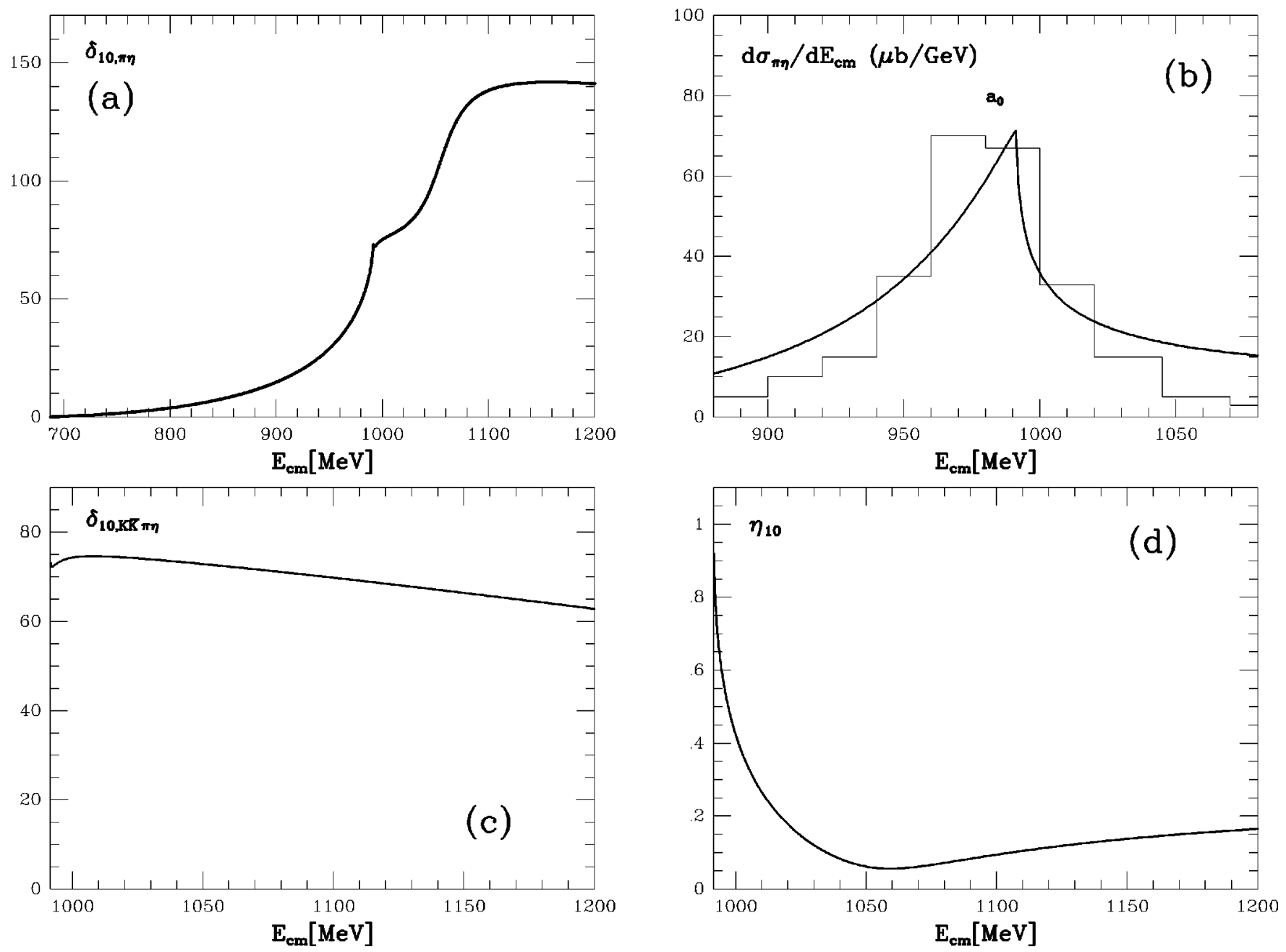

FIG. 4. Results in the $I=1, J=0$ channel. (a) Phase shifts for $\pi \eta \rightarrow \pi \eta$. (b) Invariant mass distribution for $\pi \eta$ data from [34]. (c) Phase shifts for $K \bar{K} \rightarrow \pi \eta$. (d) Inelasticity.

where $m$ is the $\pi^{-} \eta$ invariant mass, $q$ the $\pi$ momentum in the $\pi^{-} \eta$ c.m. frame, $t$ the $\pi^{-} \eta \rightarrow \pi^{-} \eta$ scattering amplitude, and $C$ a normalization constant. We observe a fairly good agreement with the experimental numbers.

\section{Channel $(1 / 2,0)$}

The two coupled states are now $K \pi$ and $K \eta$. In Fig. 5(a) we plot the phase shifts for $K \pi \rightarrow K \pi$. The theoretical curve follows the same trend as the experimental data, although it lies a bit above them. The results and the data show a broad bump, which is related to the presence of a pole which appears around $770-i 250 \mathrm{MeV}$. Such a resonance, whose existence has been claimed in a recent data analysis [42], is predicted in quark models of $q^{2} \bar{q}^{2}$ systems [41] and is usually denoted by $\kappa(900)$. This resonance bears some similarity with the $\sigma$ in the $(0,0) \pi \pi$ elastic scattering channel, which is also very broad. Finally, the $K \eta \rightarrow K \eta$ phase shifts are small as shown in Fig. 5(c) and the inelasticities given in
Fig. 5(d) are not distant from unity. This fact indicates a small mixture of $K \pi$ with $K \eta$.

\section{Channel $(1 / 2,1)$}

In this case we also find a resonance in Fig. 6(a), analogous to the $\rho$, but in the $K \pi$ system. This resonant state, known as the $K^{*}(892)$, is as clean as the $\rho$, and the agreement of our results with the data is remarkably good over the whole range of energies up to $1.2 \mathrm{GeV}$. In Fig. 6(c) we plot the $K \eta \rightarrow K \eta$ phase shifts, which are very small. Finally, in Fig. 6(d) we can notice that $\eta \approx 1$ which means that there is practically no mixture of $K \pi$ and $K \eta$ in this channel. This justifies the success of [4] reproducing this resonance using only the $K \pi$ state and elastic unitarity.

\section{Channel $(3 / 2,0)$}

In Fig. 7 we show the $K \pi$ phase shifts. As we can see in the figure, the agreement with the data is quite good up to about $1.2 \mathrm{GeV}$. 

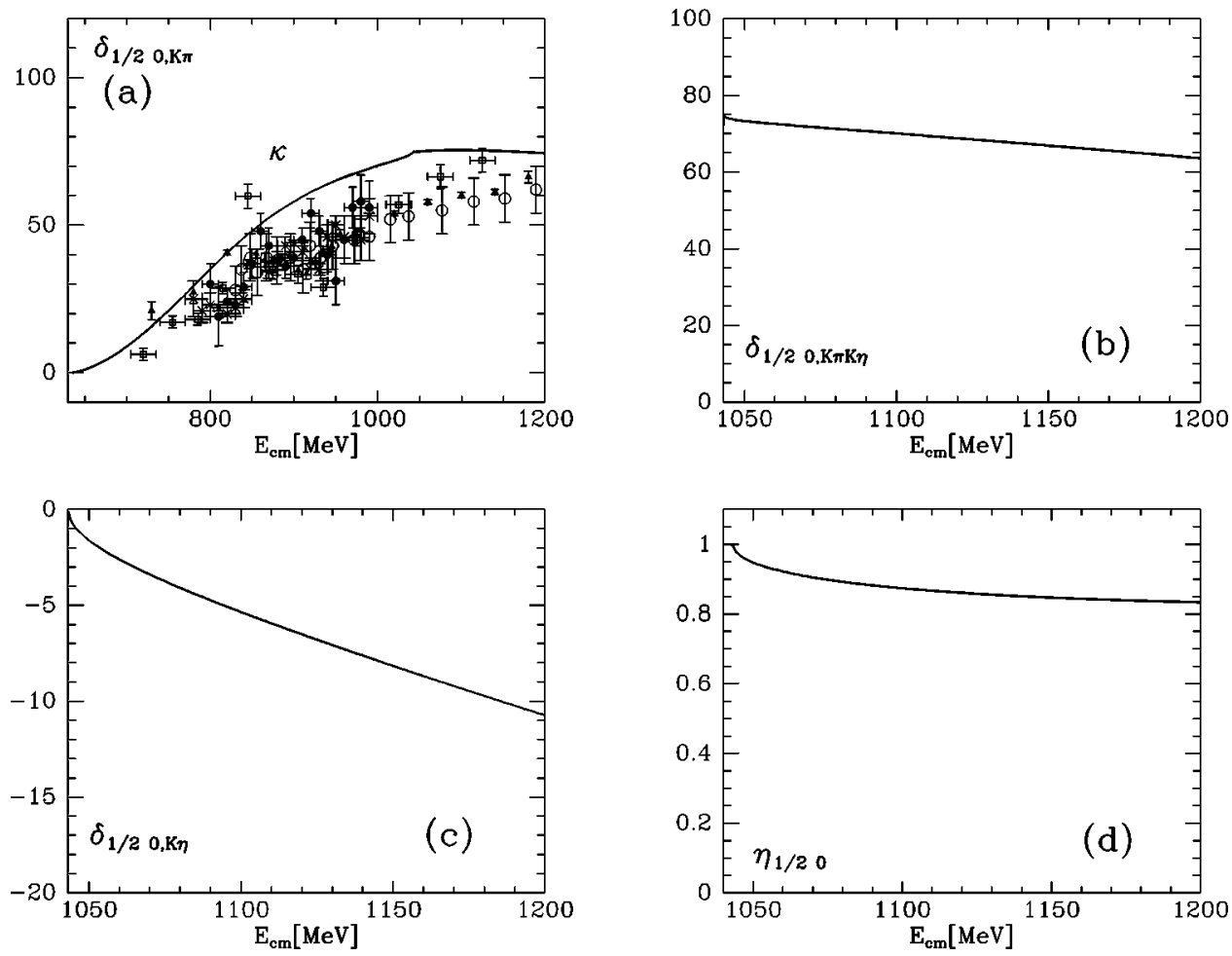

FIG. 5. Results in the $I=1 / 2, J=0$ channel. (a) Phase shifts for $K \pi \rightarrow K \pi$. Data: solid circle [36], cross [37], open square [38], solid triangle [40], open circle [39]. (b) Phase shifts for $K \pi \rightarrow K \eta$. (c) Phase shifts for $K \eta \rightarrow K \eta$. (d) Inelasticity.

The channel $(3 / 2,1)$ in $K \pi$ (see Table I) is such that $T_{2}$ $=0$, since there is only an $S$ wave there. In this case our method cannot be applied, as discussed above, and we should just take the $T_{4}$ contribution. That also happens for the $J=2$ channels, since the structure of $T_{2}$, which is $\mathcal{O}\left(p^{2}\right)$, is a linear combination of $s, t, u$ and squared masses. Therefore there is only $J=0,1$ in $T_{2}$, but not $J=2$. Hence, the lowest contribution can only be obtained from the $T_{4}$ terms and our method has nothing to improve there with respect to $\chi \mathrm{PT}$. The phase shifts in these channels are small and have been discussed in [4]. Hence we omit any further discussion, simply mentioning that the agreement with data found in [4] is fairly good.

There is another interesting result in the $(0,1)$ channel which is the appearance of a pole around $990 \mathrm{MeV}$, which we show in Fig. 8. Below $1.2 \mathrm{GeV}$ there are two resonances with such quantum numbers. They are the $\omega$ and the $\phi$, which fit well within the $q \bar{q}$ scheme, with practically ideal mixing, as $(1 / \sqrt{2})(u \bar{u}+d \bar{d})$ and $s \bar{s}$, respectively. In the limit of exact SU(3) symmetry these resonances manifest as one antisymmetric octet state and a symmetric singlet state. Since the spatial function of the $K \bar{K}$ state is antisymmetric, its $\mathrm{SU}(3)$ wave function also has to be antisymmetric and therefore it only couples to the antysimmetric octet resonance. Of course, our Lagrangians do contain some SU(3) breaking, but in this channel we are only dealing with the $K \bar{K}$ state, neglecting states with other mesons (such as the three-pion channel) and, hence, our formulas for this process do not contain any $\mathrm{SU}(3)$ symmetry breaking term. Thus, we just see one pole, corresponding to the antisymmetric octet state of the exact $\mathrm{SU}(3)$ limit. Because the ideal mixing angle is around $20^{\circ}$, the pole we obtain should be closer to the physical $\phi(1020)$ than to the physical $\omega(782)$. This is in fact what we obtain since the mass of our pole is $990 \mathrm{MeV}$, much closer to the mass of the $\phi(1020)$ meson than to the mass of the $\omega(782)$. It seems then plausible that the small coupling to three pions (an OZI suppressed coupling of third class) which we are not taking into account, could be enough to bring our pure octet state to the physical $\phi$ resonance.

\section{B. Pole positions, widths, and partial decay widths}

We will now look for the poles of the $T$ matrix in the complex plane, which should appear in the unphysical Riemann sheets (the conventions taken are those of [8], which can be easily induced from the analytical expressions of Appendix A). Let us remember that the mass and the width of a Breit-Wigner resonance are related to the position of its complex pole by $\sqrt{s_{\text {pole }}} \simeq M-i \Gamma / 2$, but this formula does not hold for other kinds of resonances. In Table III we give the results for the pole positions as well as the apparent or "effective" masses and widths that can be estimated from phase shifts and mass distributions in scattering processes. Note that such "effective" masses and widths depend on the physical process.

We shall make differentiation between the $\rho$ and $K^{*}$, which are clean elastic Breit-Wigner resonances, and the rest. For the $\rho$ and $K^{*}$ their mass is given by the energy at which $\delta=90^{\circ}$ and the width is taken from the phase shifts 

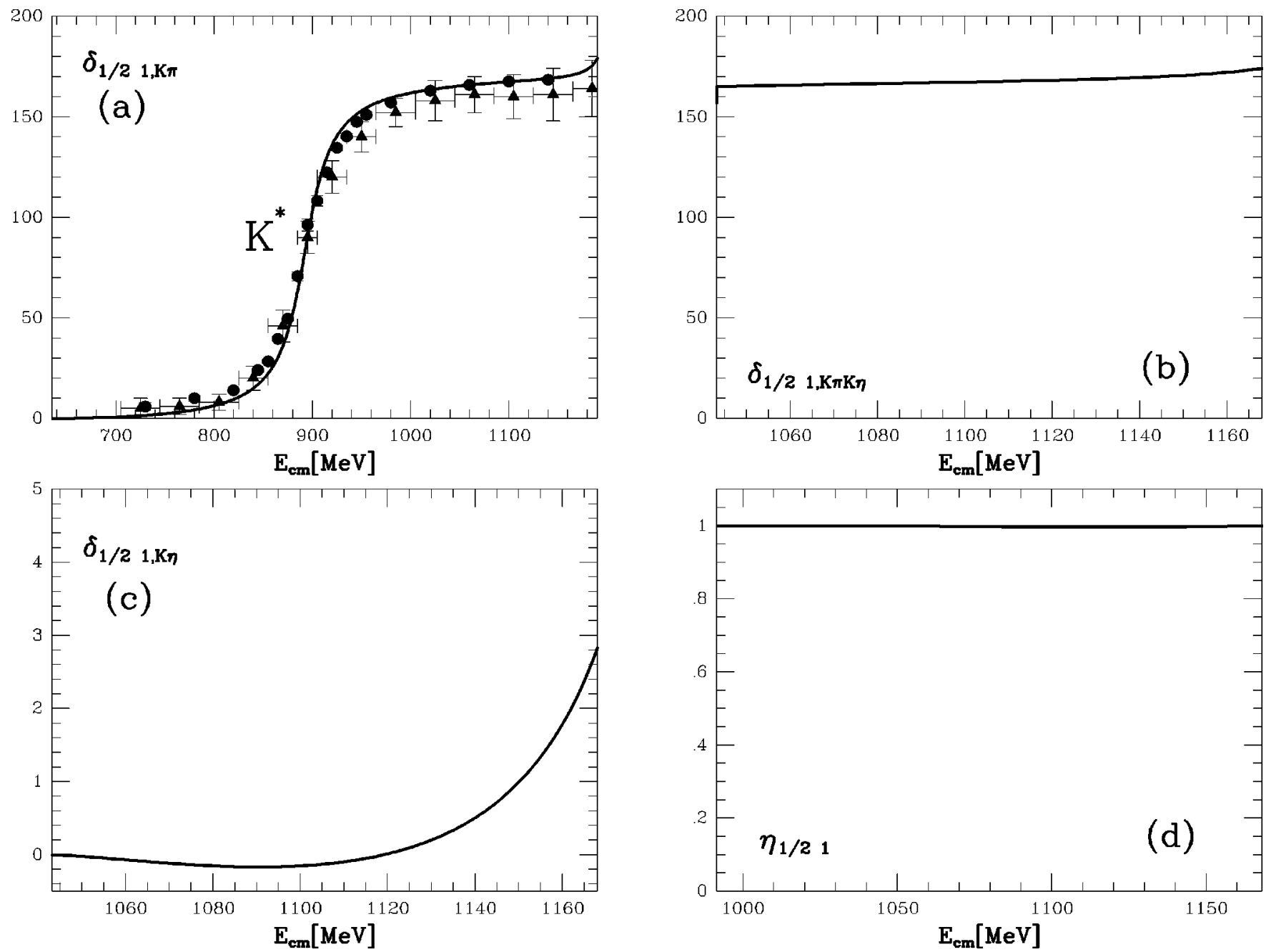

FIG. 6. Results in the $I=1 / 2, J=1$ channel. (a) Phase shifts for $K \pi \rightarrow K \pi$. Data: solid triangle [36], open circle [40]. (b) Phase shifts for $K \pi \rightarrow K \eta$. (c) Phase shifts for $K \eta \rightarrow K \eta$. (d) Inelasticity.

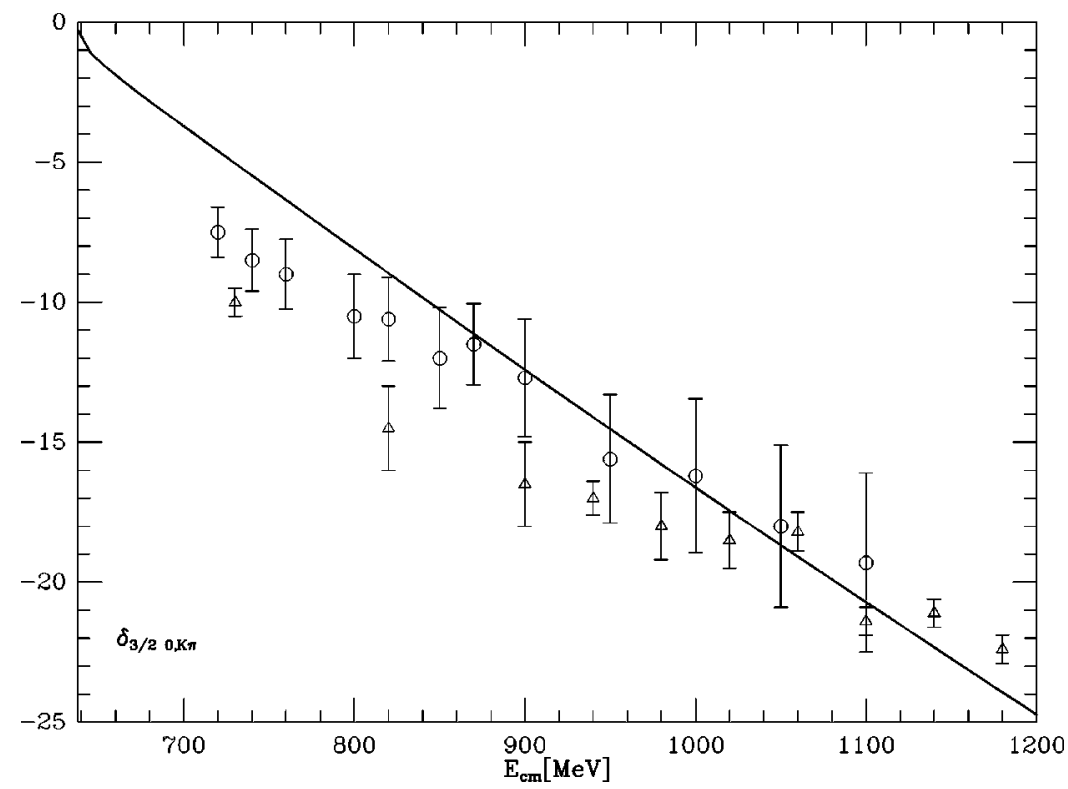

FIG. 7. Phase shifts for $K \pi \rightarrow K \pi$ in the $I=3 / 2, J=0$ channel. Data: open triangle [40], open circle [43]. 


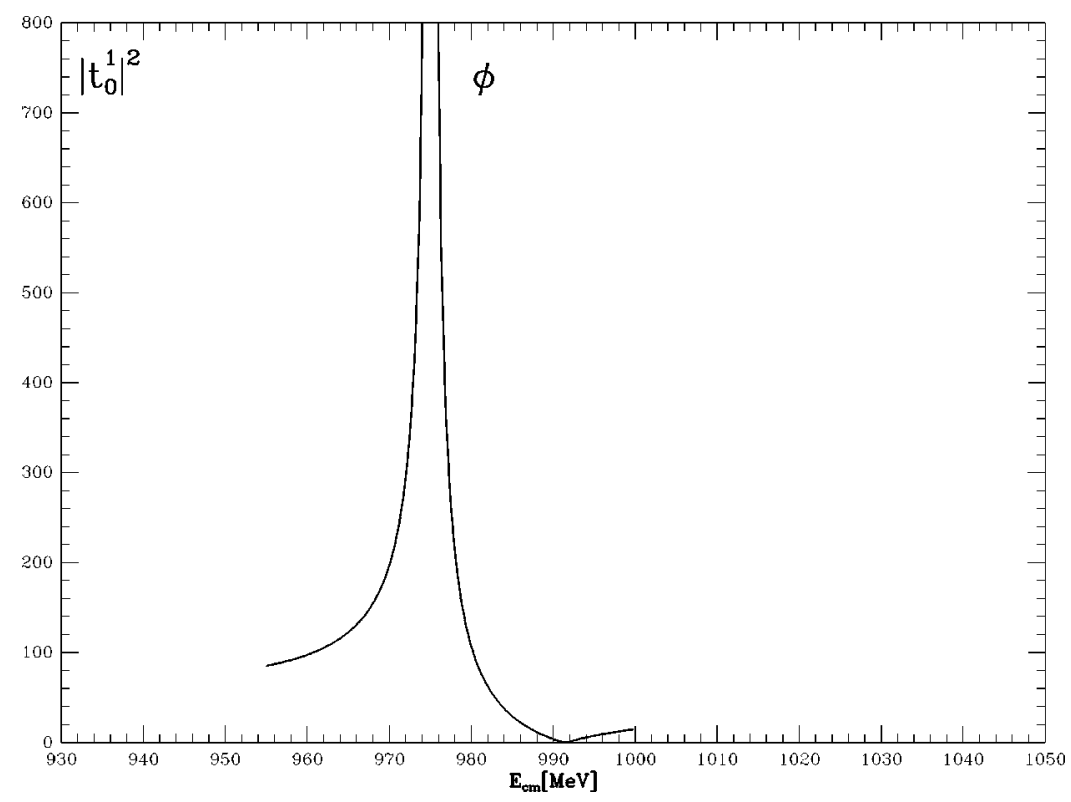

FIG. 8. $\left(\left|T_{I J=01}\right|\right)^{2}$ for $K \bar{K} \rightarrow K \bar{K}$ showing the singularity corresponding to the $\phi$ resonance.

slope around $\delta=90^{\circ}$, by means of

$$
\Gamma_{R}=\frac{M_{R}^{2}-s}{M_{R}} \tan \delta(s) .
$$

We also saw that, in practice, the $\rho$ and $K^{*}$ only couple to $\pi \pi$ and $K \pi$, respectively. The $\sigma$ decays only to $\pi \pi$ and the $\kappa$ only to $K \pi$ due to phase space and dynamical suppression of other channels [see Fig. 5(d)]. The case of the $f_{0}$ and $a_{0}$ is different, since they can decay either to $\pi \pi$ or $K \bar{K}$ (the $f_{0}$ ) and $\pi \eta$ or $K \bar{K}$ (the $a_{0}$ ). In order to determine the partial decay widths of these resonances we follow the procedure of [8], where we show that, assuming a Breit-Wigner shape for the amplitudes around the resonance pole, the partial decay widths are given by

$$
\Gamma_{R, 1}=-\frac{1}{16 \pi^{2}} \int_{E_{\min }}^{E_{\max }} d E \frac{q}{E^{2}} 4 M_{R} \operatorname{Im} T_{11},
$$

$$
\Gamma_{R, 2}=-\frac{1}{16 \pi^{2}} \int_{E_{\min }}^{E_{\max }} d E \frac{q}{E^{2}} 4 M_{R} \frac{\left(\operatorname{Im} T_{21}\right)^{2}}{\operatorname{Im} T_{11}},
$$

where $E$ stands for the total c.m. energy of the meson-meson system, $q$ is the momentum of one meson in the c.m. and the labels 1,2 stand for $K \bar{K}, \pi \pi$ in the case of the $f_{0}$ and $K \bar{K}, \pi \eta$ in the case of the $a_{0}$. The masses of the final mesons are $m_{1}, m_{2}$. The upper limit in the integral, $E_{\max }$, is $\simeq M_{R}+\Gamma_{R}$ where $\Gamma_{R}$ is the total width [8] and $E_{\min }=M_{R}$ $-\Gamma_{R}$, unless the threshold energy $\left(m_{1}+m_{2}\right)$ for the decay is bigger than that quantity, in which case $E_{\min }=m_{1}+m_{2}$. In this way we largely avoid the contribution of the backgrounds in the amplitudes. One caveat must be raised concerning Eq. (38), which was already pointed out in the study of the $f_{0} \rightarrow \gamma \gamma$ decay [44]. The subtlety is that around this resonance the phase shifts [see Fig. 1(a)] are of the order of

\begin{tabular}{|c|c|c|c|c|c|c|}
\hline $\begin{array}{l}\text { Channel } \\
(I, J)\end{array}$ & Resource & $\begin{array}{c}\text { Mass } \\
\text { from pole }\end{array}$ & $\begin{array}{l}\text { Width } \\
\text { from pole }\end{array}$ & $\begin{array}{l}\text { Mass } \\
\text { effective }\end{array}$ & $\begin{array}{l}\text { Width } \\
\text { effective }\end{array}$ & $\begin{array}{l}\text { Partial } \\
\text { widths }\end{array}$ \\
\hline$(0,0)$ & $\sigma$ & 442 & 454 & $\approx 600$ & very large & $\begin{array}{c}\pi \pi-100 \% \\
\pi \pi-65 \%\end{array}$ \\
\hline$(0,0)$ & $f_{0}(980)$ & 994 & $28 \approx 980$ & $\approx 30$ & & \\
\hline$(0,1)$ & $\phi(1020)$ & 980 & 0 & 980 & 0 & $\Lambda \Lambda-5 J \%$ \\
\hline$(1 / 2,0)$ & $\kappa$ & 770 & $500 \approx 850$ & very large & $K \pi-100 \%$ & \\
\hline$(1 / 2,1)$ & $k_{*}(890)$ & 892 & $42 * 895$ & 42 & $K \pi-100 \%$ & \\
\hline$(1,0)$ & $a_{0}(980)$ & 1055 & 42 & 980 & 40 & $\pi \eta-50 \%$ \\
\hline$(1,1)$ & $\rho(770)$ & 759 & 141 & 771 & 147 & $\begin{array}{c}K \bar{K}-50 \% \\
\pi \pi=100 \%\end{array}$ \\
\hline
\end{tabular}
$90^{\circ}$, due to the background coming from the broad $\sigma$ pole.

TABLE III. Masses and partial widths in MeV. 

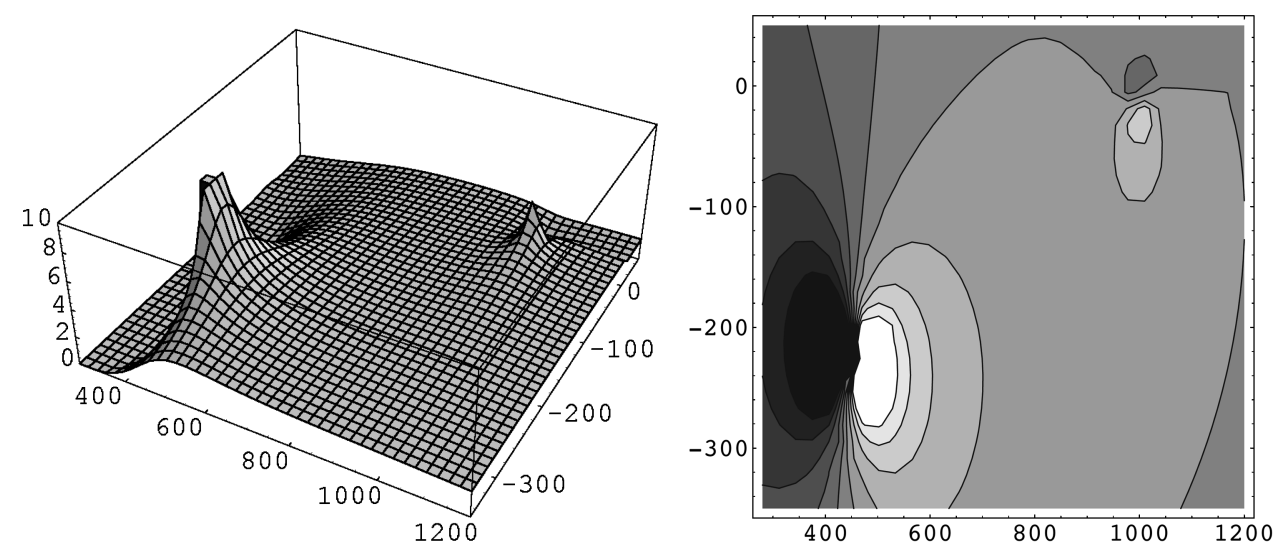

FIG. 9. Imaginary part of the $\pi \pi$ amplitude in the $(I, J)=(0,0)$ channel in the second Riemann sheet. On the left we show a threedimensional plot were we can observe the different structure of the $\sigma$ and $f_{0}$ poles. On the right we show a contour plot of the lower half plane of the second sheet. The $\sigma$ pole is very far away from the real (physical) axis and its lines of maximum gradient are parallel to it, in contrast with the $f_{0}$. That is why the effect of both poles in the phase shifts (Fig. 1) is so different.

This background causes the $f_{0} \rightarrow \pi \pi$ coupling constant to appear effectively multiplied by a $\pi / 2$ phase ( $i$ factor) and in this way the $T_{12}$ amplitude around the $f_{0}$ looks like an ordinary Breit-Wigner multiplied by $i$. This means that the real part has a peak around the resonance and the imaginary part changes sign. In this case the arguments used in [8] and [44] lead to a trivial modification in $\Gamma_{R, 2}$, where $\operatorname{Im} T_{12}$ should be substituted by $\operatorname{Re} T_{12}$.

It is also very instructing to see the representation of the poles in a three-dimensional plot. In Fig. 9 we show on the left the imaginary part of the $(0,0) \pi \pi \rightarrow \pi \pi$ scattering amplitude on the second Riemann sheet. It is possible to see very clearly the appearance of two poles that correspond to the $\sigma$ and the $f_{0}$ resonances. The former is located at 442 $-i 227$ and thus is very far away from the real axis, which implies a huge effective width. In contrast, the other pole is located at $994-i 14 \mathrm{MeV}$ accordingly to the narrow width of the $f_{0}$ resonance.

Apart from the position of the poles, there is an additional piece of information which also determines the observed shape of a resonance. It also explains some of the differences between the "effective" masses and the real part of the pole position. On the right of Fig. 9 we give a contour plot, again of the imaginary part of the $(0,0)$ amplitude in the second Riemann sheet. Notice that both poles are oriented differently, almost transversally, on the complex plane. On the one hand, the $f_{0}$ pole is oriented almost perpendicularly to the real axis, which is the relevant one in this work. As a consequence, in the positive real axis, the imaginary part of the amplitude first grows rapidly and then drops very fast again, giving rise to the dramatic variation of the phase shift typical of resonances. A similar orientation is found for the $\rho, K^{*}$, and $a_{0}$ resonances too. On the other hand, the $\sigma$ pole is oriented so that in the real axis we only see a slow and smooth increase, but almost no decrease, of the imaginary part. That is also the case of the $\kappa$ resonance. This feature, together with the fact that both the $\sigma$ and the $\kappa$ are very far from the real axis, explains why it is so hard to establish firmly their existence and their physical parameters.
Finally, in Fig. 10 we present a very detailed contour plot of the $\rho$ and $a_{0}$ poles. Both of them are almost perpendicular to the real axis, but the former is tilted clockwise, whereas the latter is tilted counterclockwise. Let us now remember that the real part of the pole position, roughly, should give us the apparent mass of the resonance. However, the lines of maximum gradient of each pole cross the real axis at a point which is slightly different from the real part of its position. Therefore, those poles rotated clockwise, as the $\rho$ or the $K^{*}$, have an apparent mass a little bit higher than that given by the pole position. In contrast those tilted counterclockwise yield a resonance whose mass is somewhat lower that the one obtained from the pole. That is the case of the $f_{0}$ and the $a_{0}$.

\section{CONCLUSIONS AND OUTLOOK}

We have used a coupled channel unitary approach, together with the dynamical information contained in the $\mathcal{O}\left(p^{2}\right)$ and $\mathcal{O}\left(p^{4}\right)$ chiral Lagrangian, which allows us to study the meson-meson interaction up to about $1.2 \mathrm{GeV}$. This nonperturbative method generates poles in the complex plane corresponding to physical resonances. We have used the experimental information available to make a fit of the $\mathcal{O}\left(p^{4}\right)$ Lagrangian coefficients. These are $\hat{L}_{i}, i$ $=1,2,3,4,5,7$ and $2 \hat{L}_{6}+\hat{L}_{8}$, whose actual values depend on the cutoff that we have used to regularize divergent one-loop integrals. With those seven degrees of freedom we are able to fit, up to $1.2 \mathrm{GeV}$, all the experimental information in seven meson-meson channels. Each one of these channels consists of two phase shifts and an inelasticity. Moreover, in our results, we obtain the position and widths, partial decay widths, etc. of all the resonances that appear in those channels below $1.2 \mathrm{GeV}$. Apart from the standard $f_{0}, a_{0}, \rho, K^{*}$ resonances, we find poles in the $T$ matrix for the $\sigma$ in the $\pi \pi I=J=0$ channel and for $\kappa$ in the $(1 / 2,0)$ channel, both of them very broad. 

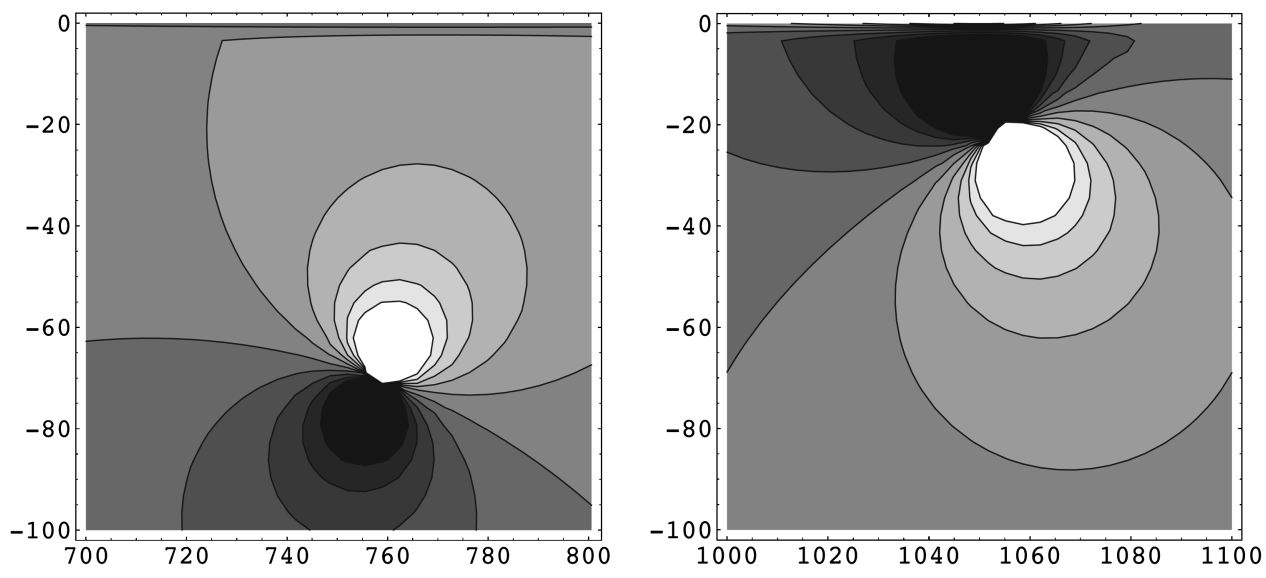

FIG. 10. The poles associated with the $\rho$ (left) and $a_{0}$ (right) are oriented differently. The $\rho$ mass seen on the $(I, J)=(1,1)$ phase shifts is slightly bigger than the real part of the position of the $\rho$ pole, whereas the peak of the mass distribution where the $a_{0}$ is observed (see Fig. 4) is smaller than the real part of the $a_{0}$ pole. Concerning the widths, they are obtained as twice the imaginary part of the associated pole position.

The method has proved very efficient to extend the ideas of chiral symmetry at energies beyond the realm of applicability of $\chi$ PT. However, at energies higher than $1.2 \mathrm{GeV}$, the limitations of the model show up, since, among other things, we have restricted ourselves only to two meson states. The restrictions in the space of states precluded the appearance of the $\omega$ resonance which couples dominantly to three pions. However, the $\phi$ resonance which couples strongly to $K \bar{K}$ does appear in the scheme, although slightly shifted towards lower energies. Presumably, by including the $\phi$ coupling to three pions, although very small, it should be enough to shift the mass to its correct place.

One of the formal weaknesses of the approach is that loops in crossed channels, as well as some tadpole contributions, are not explicitly included in the calculation. In practice, their effect can be reabsorbed in the fit of the $\mathcal{O}\left(p^{4}\right)$ parameters, whose values can then be different from those obtained for the standard low energy $\chi \mathrm{PT}$ approach.

This approximation could be improved by using Eq. (13) with the full $\mathcal{O}\left(p^{4}\right) \chi \mathrm{PT}$ calculation, which includes one loop in crossed channels and the tadpoles. This would allow a more straightforward comparison with standard $\chi \mathrm{PT}$ as well as a better accuracy in the low energy results. Although such calculations are welcome and there is indeed some work in progress [45], they are far more involved to calculate and use.

Applications of the method to other physical problems are also in order. Indeed, it can be easily extended to deal with processes where meson pairs appear in the initial or final state, like meson pair photoproduction [44]. It looks likely that it could also prove useful describing the meson-nucleon interaction [46] complemented with heavy baryon chiral perturbation theory. In addition, the method, nonperturbative in nature, is equally well suited to study the meson-meson interaction in a nuclear medium where there has been some speculation about the appearance of bound $\pi \pi$ pairs [47].

Finally it seems that the approach could be extended to the effective chiral Lagrangian description of the standard model strongly interacting symmetry breaking sector, where the single channel approach has already been applied [48].

\section{ACKNOWLEDGMENTS}

We are grateful to A. Dobado for discussions concerning the tadpole contributions and for his careful reading of the manuscript. Two of us, J.A.O and E.O., would like to thank the kind hospitality of the Complutense University of Madrid. J.R.P. wishes to thank the hospitality of the University of Valencia and the SLAC Theory Group, as well as M. Suzuki for his comments on the octet $\phi$. This work was partially supported by DGICYT under contracts PB96-0753 and AEN93-0776. J.A.O. and J.R.P. acknowledge financial support from the Generalitat Valenciana and the Ministerio de Educación y Cultura, respectively.

\section{APPENDIX A: ANALYTICAL FORMULA FOR $G(s)$ : RELATION BETWEEN CUTOFF AND DIMENSIONAL RENORMALIZATION}

In this appendix we show the relationship between our regularization scheme and dimensional regularization, which is the usual one when dealing with $\chi \mathrm{PT}$.

\section{Analytical formula for $G(s)$ with a cutoff regularization}

In the general case with different masses $M_{1}$ and $M_{2}$, 


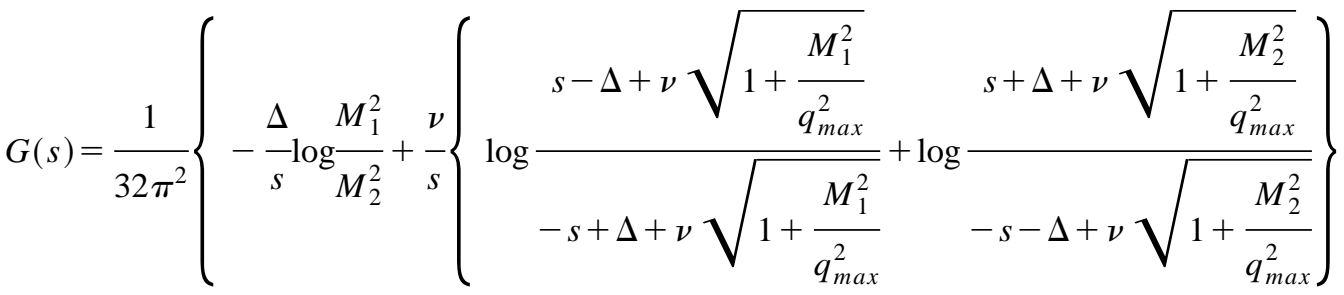

$$
\begin{aligned}
& \left.+2 \frac{\Delta}{s} \log \frac{1+\sqrt{1+\frac{M_{2}^{2}}{q_{\text {max }}^{2}}}}{1+\sqrt{1+\frac{M_{2}^{2}}{q_{\text {max }}^{2}}}}-2 \log \left[\left(1+\sqrt{1+\frac{M_{1}^{2}}{q_{\text {max }}^{2}}}\right)\left(1+\sqrt{1+\frac{M_{2}^{2}}{q_{\max }^{2}}}\right)\right]+\log \frac{M_{1}^{2} M_{2}^{2}}{q_{\max }^{4}}\right\},
\end{aligned}
$$

where $\nu=\sqrt{\left[s-\left(M_{1}+M_{2}\right)^{2}\right]\left[s-\left(M_{1}-M_{2}\right)^{2}\right]}$ and $\Delta=M_{1}^{2}-M_{2}^{2}$. In the case of equal masses $M_{1}=M_{2}=m$, the above formula reduces to

$$
G(s)=\frac{1}{(4 \pi)^{2}}\left[\begin{array}{c}
\sigma \sqrt{1+\frac{m^{2}}{q_{\max }^{2}}}+1 \\
\sigma \log \frac{m_{1+\frac{m^{2}}{q_{\text {max }}^{2}}}}{\sigma}-1
\end{array}-2 \log \left\{\frac{q_{\max }}{m}\left(1+\sqrt{1+\frac{m^{2}}{q_{\text {max }}^{2}}}\right)\right\},\right.
$$

where now, $\sigma=\sqrt{1-4 m^{2} / s}$.

The numerical evaluation of the principal part of Eq. (15) is also performed as an additional check.

\section{Relation between the cutoff and the dimensional regularization scale}

In order to obtain the relationship between the cutoff and the renormalization scale $\mu$ let us consider, for the sake of simplicity, the case with equal masses (the same result is obtained with different masses but the formulas are more cumbersome). As far as we are going to compare the same function calculated in different ways, let us denote by $G^{C}(s)$ the $G(s)$ calculated with a cutoff regularization and $G^{D}(s)$ the one calculated with dimensional regularization. In this latter case we have

$$
G^{D}(s)=\frac{1}{(4 \pi)^{2}}\left[\frac{1}{\hat{\epsilon}}-2+\log m^{2}+\sigma \log \frac{\sigma+1}{\sigma-1}\right],
$$

where $1 / \hat{\epsilon}=1 / \epsilon-\log (4 \pi)+\gamma$ with $D=4+2 \epsilon$.

The scale $\mu$ in $G^{D}(s)$ appears through the inclusion of the $L_{i}$ [2] at $\mathcal{O}\left(p^{4}\right)$ :

$$
L_{i}=L_{i}^{r}(\mu)+\Gamma_{i} \lambda,
$$

where $L_{i}^{r}(\mu)$ is the renormalized value of $L_{i}$ at the $\mu$ scale, $\Gamma_{i}$ is just a number, and

$$
\lambda=\frac{1}{32 \pi^{2}}\left[\frac{1}{\hat{\epsilon}}+\log \mu^{2}-1\right] .
$$

The $\log \mu^{2}$, and its companion $1 / \hat{\epsilon}-1$ are incorporated in $G^{D}(s)$ so that at the end one has a logarithm of the dimensionless quantity $m^{2} / \mu^{2}$. In this way we rewrite $G^{D}(s)$ as

$$
G^{D}(s)=\frac{1}{(4 \pi)^{2}}\left[-1+\log \frac{m^{2}}{\mu^{2}}+\sigma \log \frac{\sigma+1}{\sigma-1}\right] .
$$

We expand Eq. (A2) in powers of $m^{2} / q_{\max }^{2}$ to compare with the cutoff regularization, as follows: 


$$
\begin{aligned}
G^{C}(s) & =\frac{1}{(4 \pi)^{2}}\left[-2 \log \frac{2 q_{\text {max }}}{m}+\sigma \log \frac{\sigma+1}{\sigma-1}+\mathcal{O}\left(\frac{m^{2}}{q_{\text {max }}^{2}}\right)\right] \\
& =\frac{1}{(4 \pi)^{2}}\left[-1+\log e+\log \frac{m^{2}}{4 q_{\text {max }}^{2}}+\sigma \log \frac{\sigma+1}{\sigma-1}+\mathcal{O}\left(\frac{m^{2}}{q_{\text {max }}^{2}}\right)\right] \\
& =\frac{1}{(4 \pi)^{2}}\left[-1+\log \frac{m^{2} e}{4 q_{\text {max }}^{2}}+\sigma \log \frac{\sigma+1}{\sigma-1}+\mathcal{O}\left(\frac{m^{2}}{q_{\text {max }}^{2}}\right)\right] .
\end{aligned}
$$

Then comparing Eqs. (A6) and (A7) one has:

$$
\mu=\frac{2 q_{\text {max }}}{\sqrt{e}} \simeq 1.2 q_{\text {max }} .
$$

Hence, to our cutoff $q_{\text {max }} \simeq 1 \mathrm{GeV}$ would correspond a $\mu=1.2 \mathrm{GeV}$ dimensional regularization scale. In Table II, we have listed the values of the $\hat{L}_{i}$ parameters and those of standard $\chi$ PT scaled to $\mu=1.2 \mathrm{GeV}$. As it is explained in the text, in our fit we have neglected the crossed channel diagrams and we have treated tadpoles differently. The effect of these contributions is effectively reabsorbed in our $\hat{L}_{i}$ parameters; hence some differences between the $\hat{L}_{i}$ and $L_{i}$ parameters should be expected and this is indeed the case as can be seen in Table II. Note that, even if we had used the complete $\mathcal{O}\left(p^{4}\right) \chi \mathrm{PT}$ calculations, these parameters could be different, since they have been obtained from a fit over a much wider range of energies than it is used in $\chi \mathrm{PT}$ and higher order contributions have been included.

Finally, note that the terms $\mathcal{O}\left(\mathrm{m}^{2} / q_{\text {max }}^{2}\right)$ in Eq. (A7) yield $\mathcal{O}\left(p^{6}\right)$, or higher, contributions and that is why they are not included in $G^{D}(s)$.

It is also worth stressing that the relationship of Eq. (A8) is independent of the physical process and channel since the function $G(s)$ appears in all them in the same way.

\section{APPENDIX B: AMPLITUDES}

We have used the following formulas in our calculations. Note that, as has been explained in the text, we have an overall sign of difference with the definitions in [1], as well as a $1 / 2$ factor for those amplitudes with identical particles.

$$
\begin{gathered}
\text { 1. Masses and decay constants } \\
f_{\pi}=f_{0}\left[1+\frac{4 m_{\pi}^{2}}{f_{0}^{2}}\left(L_{5}+L_{4}\right)+\frac{8 m_{K}^{2}}{f_{0}^{2}} L_{4}\right], \\
f_{K}=f_{0}\left[1+\frac{4 m_{K}^{2}}{f_{0}^{2}}\left(L_{5}+2 L_{4}\right)+\frac{4 m_{\pi}^{2}}{f_{0}^{2}} L_{4}\right], \\
f_{\eta}=f_{0}\left[1+\frac{4 m_{\eta}^{2}}{f_{0}^{2}} L_{5}+\frac{8 m_{K}^{2}+4 m_{\pi}^{2}}{f_{0}^{2}} L_{4}\right],
\end{gathered}
$$

$$
\begin{aligned}
m_{\pi}^{2}= & m_{0 \pi}^{2}\left[1+\frac{8 m_{\pi}^{2}}{f_{0}^{2}}\left(2 L_{6}+2 L_{8}-L_{4}-L_{5}\right)\right. \\
& \left.+\frac{16 m_{K}^{2}}{f_{0}^{2}}\left(2 L_{6}-L_{4}\right)\right], \\
m_{K}^{2}= & m_{0 K}^{2}\left[1+\frac{16 m_{K}^{2}}{f_{0}^{2}}\left(2 L_{6}+L_{8}-L_{4}-\frac{1}{2} L_{5}\right)\right. \\
& \left.+\frac{8 m_{\pi}^{2}}{f_{0}^{2}}\left(2 L_{6}-L_{4}\right)\right],
\end{aligned}
$$

where the 0 subscript refers to bare quantities.

\section{2. $\pi \pi \rightarrow \pi \pi$ scattering}

The definite isospin amplitudes $T^{(I)}$ are obtained from just one amplitude $T$ :

$$
\begin{aligned}
& T^{(0)}(s, t, u)=[3 T(s, t, u)+T(t, s, u)+T(u, t, s)] / 2, \\
& T^{(1)}(s, t, u)=[T(t, s, u)-T(u, t, s)] / 2, \\
& T^{(2)}(s, t, u)=[T(t, s, u)+T(u, t, s)] / 2,
\end{aligned}
$$

where $T=T_{2}+T_{4}$ is given by

$$
\begin{aligned}
T_{2}= & \frac{m_{\pi}^{2}-s}{f_{\pi}^{2}}, \\
T_{4}= & -\frac{4}{f_{\pi}^{4}}\left\{\left(2 L_{1}+L_{3}\right)\left(s-2 m_{\pi}^{2}\right)^{2}+L_{2}\left[\left(t-2 m_{\pi}^{2}\right)^{2}\right.\right. \\
& \left.+\left(u-2 m_{\pi}^{2}\right)^{2}\right]+2\left(2 L_{4}+L_{5}\right) m_{\pi}^{2}\left(s-2 m_{\pi}^{2}\right) \\
& \left.+4\left(2 L_{6}+L_{8}\right) m_{\pi}^{4}\right\},
\end{aligned}
$$

which have been obtained at the tree level from $\mathcal{L}_{2}$ and $\mathcal{L}_{4}$, respectively. 


\section{3. $K \pi \rightarrow K \pi$ scattering}

Using crossing symmetry, we can write the $I=1 / 2$ amplitude in terms of that with $I=3 / 2$, as

$$
T^{(1 / 2)}(s, t, u)=\frac{3}{2} T^{(3 / 2)}(u, t, s)-\frac{1}{2} T^{(3 / 2)}(s, t, u),
$$

where

$$
\begin{aligned}
T_{2}^{(3 / 2)}= & \frac{s-\left(m_{\pi}^{2}+m_{K}^{2}\right)}{2 f_{\pi} f_{K}}, \\
T_{4}^{(3 / 2)}= & -\frac{2}{f_{\pi}^{2} f_{K}^{2}}\left\{\left(4 L_{1}+L_{3}\right)\left(t-2 m_{\pi}^{2}\right)\left(t-2 m_{K}^{2}\right)+2 L_{2}\left(m_{\pi}^{2}+m_{K}^{2}-s\right)^{2}\right. \\
& +\left(2 L_{2}+L_{3}\right)\left(m_{\pi}^{2}+m_{K}^{2}-u\right)^{2}+4 L_{4}\left[\left(m_{\pi}^{2}+m_{K}^{2}\right) t-4 m_{\pi}^{2} m_{K}^{2}\right] \\
& \left.+L_{5}\left[\left(m_{\pi}^{2}+m_{K}^{2}\right)\left(m_{\pi}^{2}+m_{K}^{2}-s\right)-4 m_{\pi}^{2} m_{K}^{2}\right]+8 m_{\pi}^{2} m_{K}^{2}\left(2 L_{6}+L_{8}\right)\right\},
\end{aligned}
$$

which, once more, have been obtained, respectively, from $\mathcal{L}_{2}$ and $\mathcal{L}_{4}$ at the tree level.

\section{4. $K \bar{K} \rightarrow K \bar{K}$ scattering}

The definite isospin amplitudes can be written just in terms of two:

$$
\begin{aligned}
& T^{(0)}(s, t, u)=T^{+-+-}(s, t, u)+T^{\overline{0} 0+-}(s, t, u), \\
& T^{(1)}(s, t, u)=T^{+-+-}(s, t, u)-T^{\overline{0} 0+-}(s, t, u),
\end{aligned}
$$

where $T^{+-+-}$is the amplitude for $K^{+} K^{-} \rightarrow K^{+} K^{-}$, whose respective $\mathcal{O}\left(p^{2}\right)$ and $\mathcal{O}\left(p^{4}\right)$ contributions are

$$
\begin{aligned}
& T_{2}^{+-+-}(s, t, u)=\frac{u-2 m_{K}^{2}}{f_{K}^{2}}, \\
& T_{4}^{,+-+-}(s, t, u)=-\frac{4}{f_{K}^{4}}\left\{2 L_{2}\left(u-2 m_{K}^{2}\right)^{2}+\left(2 L_{1}+L_{2}+L_{3}\right)\left[\left(s-2 m_{K}^{2}\right)^{2}+\left(t-2 m_{K}^{2}\right)^{2}\right]-2 u m_{K}^{2}\left(2 L_{4}+L_{5}\right)+8 m_{K}^{4}\left(2 L_{6}+L_{8}\right)\right\}
\end{aligned}
$$

whereas $T^{\overline{0} 0+-}$ is the amplitude for $\bar{K}^{0} K^{0} \rightarrow K^{+} K^{-}$, which is given by

$$
\begin{aligned}
T_{2}^{\overline{0} 0+-}(s, t, u)= & \frac{u-2 m_{K}^{2}}{2 f_{K}^{2}}, \\
T_{4}^{\overline{00+-}}(s, t, u)= & -\frac{2}{f_{K}^{4}}\left\{\left(4 L_{1}+L_{3}\right)\left(s-2 m_{K}^{2}\right)^{2}+\left(2 L_{2}+L_{3}\right)\left(t-2 m_{K}^{2}\right)^{2}+2 L_{2}\left(u-2 m_{K}^{2}\right)^{2}\right. \\
& \left.+2 s m_{K}^{2}\left(4 L_{4}+L_{5}\right)+\frac{2}{3} t m_{K}^{2}\left(2 L_{4}+L_{5}\right)-8 m_{K}^{4}\left(2 L_{4}+L_{5}-2 L_{6}-L_{8}\right)\right\} .
\end{aligned}
$$




\section{a. $\pi \pi \rightarrow K \bar{K}$ scattering}

Again, we can use crossing symmetry to obtain, from $K \pi \rightarrow K \pi$, the definite isospin amplitudes $T_{I}$ of this process:

$$
\begin{aligned}
& T^{(0)}=\frac{\sqrt{3}}{2}\left[T^{(3 / 2)}(u, s, t)+T^{(3 / 2)}(t, s, u)\right], \\
& T^{(1)}=\frac{1}{\sqrt{2}}\left[T^{(3 / 2)}(u, s, t)-T^{(3 / 2)}(t, s, u)\right] .
\end{aligned}
$$

\section{b. $K \boldsymbol{\eta} \rightarrow K \boldsymbol{\eta}$ scattering}

This process is pure $I=1 / 2$. We obtain the following contributions to the amplitude:

$$
\begin{aligned}
T_{2}(s, t, u)= & \frac{6 m_{\eta}^{2}+2 m_{\pi}^{2}-9 t}{12 f_{\eta} f_{K}} \\
T_{4}(s, t, u)= & -\frac{1}{3 f_{K}^{2} f_{\eta}^{2}}\left\{2\left(t-2 m_{K}^{2}\right)\left(t-2 m_{\eta}^{2}\right)\left(12 L_{1}+5 L_{3}\right)+\left[\left(u-m_{\eta}^{2}-m_{K}^{2}\right)^{2}+\left(s-m_{\eta}^{2}-m_{K}^{2}\right)^{2}\right]\right. \\
& \times\left(12 L_{2}+L_{3}\right)+2\left(t-2 m_{K}^{2}\right)\left[11 m_{K}^{2}\left(2 L_{4}+L_{5}\right)-m_{\pi}^{2}\left(L_{4}+3 L_{5}\right)\right] \\
& +4 m_{K}^{4}\left[3\left(2 L_{4}+L_{5}\right)+32\left(L_{6}+L_{7}+L_{8}\right)\right]+2\left(t-2 m_{\eta}^{2}\right)\left[9 m_{K}^{2}\left(2 L_{4}+L_{5}\right)+m_{\pi}^{2}\left(3 L_{4}-L_{5}\right)\right] \\
& +4 m_{\pi}^{4}\left(16 L_{7}+8 L_{8}-L_{5}\right)+6 L_{5} m_{\eta}^{4}-t\left[m_{K}^{2}\left(24 L_{4}+7 L_{5}\right)+2 m_{\pi}^{2}\left(6 L_{4}-L_{5}\right)+9 L_{5} m_{\eta}^{2}\right] \\
& \left.+6 m_{\eta}^{2} m_{K}^{2}\left(4 L_{4}+L_{5}\right)+2 m_{\eta}^{2} m_{\pi}^{2}\left(6 L_{4}+L_{5}\right)+2 m_{K}^{2} m_{\pi}^{2}\left[6 L_{4}+L_{5}-8\left(2 L_{6}+7 L_{8}+12 L_{7}\right)\right]\right\} . \\
& \quad \boldsymbol{c .} \boldsymbol{K} \boldsymbol{\eta} \rightarrow \boldsymbol{K} \boldsymbol{\pi} \boldsymbol{s c a t t e r i n g}
\end{aligned}
$$

The $I=1 / 2$ amplitude can be obtained as follows:

$$
T^{(1 / 2)}(s, t, u)=\sqrt{\frac{3}{2}} T_{\bar{K}^{0} \eta \rightarrow K^{-} \pi^{+}}(s, t, u) .
$$

The $\mathcal{O}\left(p^{2}\right)$ and $\mathcal{O}\left(p^{4}\right)$ contributions to $\bar{K}^{0} \eta \rightarrow K^{-} \pi^{+}$are

$$
\begin{aligned}
T_{2}(s, t, u)= & \frac{\sqrt{6}\left[8 m_{K}^{2}+3 m_{\eta}^{2}+m_{\pi}^{2}-9 t\right]}{36 f_{K} f_{\eta}}, \\
T_{4}(s, t, u)= & -\frac{\sqrt{2 / 3}}{3 f_{K}^{2} f_{\eta}^{2}}\left\{3 L _ { 3 } \left[2\left(t-m_{\pi}^{2}-m_{\eta}^{2}\right)\left(t-2 m_{K}^{2}\right)-\left(s-m_{K}^{2}-m_{\pi}^{2}\right)\left(s-m_{K}^{2}-m_{\eta}^{2}\right)\right.\right. \\
& \left.-\left(u-m_{K}^{2}-m_{\pi}^{2}\right)\left(u-m_{K}^{2}-m_{\eta}^{2}\right)\right]+L_{5}\left[\left(t+m_{\pi}^{2}-m_{\eta}^{2}\right)\left(7 m_{K}^{2}-5 m_{\pi}^{2}\right)\right. \\
& \left.+4 m_{K}^{2}\left(3 t-3 m_{\pi}^{2}-m_{\eta}^{2}\right)+2\left(t-2 m_{K}^{2}\right)\left(m_{K}^{2}+m_{\pi}^{2}\right)+4\left(m_{\pi}^{4}-m_{K}^{4}\right)\right] \\
& \left.+16\left(2 L_{7}+L_{8}\right)\left(m_{\pi}^{4}-2 m_{K}^{4}+m_{K}^{2} m_{\pi}^{2}\right)\right\} . \\
& \boldsymbol{d} . \boldsymbol{\eta} \boldsymbol{\pi} \rightarrow \boldsymbol{\eta} \boldsymbol{\pi}
\end{aligned}
$$

This channel is pure $I=1$ isospin. The amplitude is given by

$$
T_{2}(s, t, u)=\frac{-m_{\pi}^{2}}{3 f_{\eta} f_{\pi}}
$$




$$
\begin{aligned}
T_{4}(s, t, u)= & -\frac{4}{3 f_{K}^{2} f_{\eta}^{2}}\left\{\left(t-2 m_{\pi}^{2}\right)\left(t-2 m_{\eta}^{2}\right)\left(6 L_{1}+L_{3}\right)+4 t L_{4}\left(m_{\pi}^{2}+2 m_{K}^{2}\right)\right. \\
& +\left(3 L_{2}+L_{3}\right)\left[\left(s-m_{\pi}^{2}-m_{\eta}^{2}\right)^{2}+\left(u-m_{\pi}^{2}-m_{\eta}^{2}\right)^{2}\right]+m_{\pi}^{4}\left(4 L_{4}-L_{5}-8 L_{6}+32 L_{7}+12 L_{8}\right) \\
& \left.-16 m_{K}^{2} m_{\pi}^{2}\left(L_{4}-2 L_{6}+2 L_{7}\right)-3 m_{\pi}^{2} m_{\eta}^{2}\left(4 L_{4}+L_{5}\right)\right\} .
\end{aligned}
$$

[1] J. Gasser and H. Leutwyler, Ann. Phys. (N.Y.) 158, 142 (1984); Nucl. Phys. B250, 465 (1985).

[2] U.G. Meissner, Rep. Prog. Phys. 56, 903 (1993); V. Bernard, N. Kaiser, and U.G. Meissner, Int. J. Mod. Phys. E 4, 193 (1995); A. Pich, Rep. Prog. Phys. 58, 563 (1995); G. Ecker, Prog. Part. Nucl. Phys. 35, 1 (1995).

[3] J.V. Steele, H. Yamagishi, and I. Zahed, Nucl. Phys. A615, 305 (1997).

[4] A. Dobado, M.J. Herrero, and T.N. Truong, Phys. Lett. B 235, 134 (1990); A. Dobado and J.R. Peláez, Phys. Rev. D 47, 4883 (1993).

[5] A. Dobado and J.R. Peláez, Phys. Rev. D 56, 3057 (1997).

[6] T.N. Truong, Phys. Rev. Lett. 61, 2526 (1988); 67, 2260 (1991).

[7] M. Boglione, M.R. Pennington, Z. Phys. C 75, 113 (1997); T. Hannah, hep-ph/9703403.

[8] J.A. Oller and E. Oset, Nucl. Phys. A620, 438 (1997).

[9] G. Ecker, J. Gasser, A. Pich, and E. de Rafael, Nucl. Phys. B321, 311 (1989).

[10] J.A. Oller, E. Oset, and J.R. Peláez, Phys. Rev. Lett. 80, 3452 (1998).

[11] A.D. Martin and T.D. Spearman, Elementary Particle Theory (Wiley, New York, 1970), p. 348.

[12] J.L. Basdevant, Fortschr. Phys. 20, 283 (1972).

[13] J. Charap, Phys. Rev. D 2, 1115 (1970); I.S. Gerstein, R. Jackiw, B.W. Lee, and S. Weinberg, ibid. 3, 2486 (1971). J. Honerkamp, Nucl. Phys. B36, 130 (1972).

[14] L. Tararu, Phys. Rev. D 12, 3351 (1975).

[15] F. Guerrero and A. Pich, Phys. Lett. B 412, 382 (1997).

[16] L.V. Dung and T.N. Truong, hep-ph/9607378.

[17] J. Weinstein and N. Isgur, Phys. Rev. D 41, 2236 (1990).

[18] G. Grayer et al., Nucl. Phys. B75, 189 (1974).

[19] B. Hyams et al., Nucl. Phys. B64, 134 (1973).

[20] P. Estabrooks et al., in $\pi-\pi$ Scattering, edited by D.K. Williams and V. Hagopian, AIP Conf. Proc. 13 (AIP, New York, 1973), p. 37.

[21] G. Grayer et al., in Experimental Meson Spectroscopy, edited by A.H. Rosenfeld and K.W. Lai, AIP Conf. Proc. 8 (AIP, New York, 1972), p. 5.
[22] G. Grayer et al., in $\pi-\pi$ Scattering [21], p. 117.

[23] G. Grayer et al., presented at the 16th International Conference on High-Energy Physics, Batavia, 1972, paper No. 768.

[24] S.D. Protopopescu and M. Alson-Garnjost, Phys. Rev. D 7, 1279 (1973).

[25] W. Manner, presented at the 4th International Conference on Experimental Meson Spectroscopy, Boston, MA, 1974; CERN report.

[26] C.D. Frogatt and J.L. Petersen, Nucl. Phys. B129, 89 (1977).

[27] D. Cohen, Phys. Rev. D 22, 2595 (1980).

[28] A.D. Martin and E.N. Ozmuth, Nucl. Phys. B158, 520 (1979).

[29] S.J. Lindenbaum and R.S. Longacre, Phys. Lett. B 274, 492 (1992).

[30] P. Estabrooks and A.D. Martin, Nucl. Phys. B79, 301 (1974).

[31] L. Rosselet et al., Phys. Rev. D 15, 574 (1977).

[32] A. Schenk, Nucl. Phys. B363, 97 (1991).

[33] G. Jansen, B.C. Pearce, K. Holinde, and J. Speth, Phys. Rev. D 52, 2690 (1995).

[34] Amsterdam, CERN, Nijmegen-Oxford Collaboration, J.B. Gay et al., Phys. Lett. 63B, 220 (1976).

[35] S.M. Flatte, Phys. Lett. 63B, 224 (1976).

[36] R. Mercer et al., Nucl. Phys. B32, 381 (1971).

[37] H.H. Bingham et al., Nucl. Phys. B41, 1 (1972).

[38] S.L. Baker et al., Nucl. Phys. B99, 211 (1975).

[39] D. Aston et al., Nucl. Phys. B296, 493 (1988).

[40] P. Estabrooks et al., Nucl. Phys. B133, 490 (1978).

[41] R.L. Jaffe, Phys. Rev. D 15, 267 (1977); 15, 281 (1977).

[42] S. Ishida et al., Prog. Theor. Phys. 98, 621 (1997).

[43] D. Linglin et al., Nucl. Phys. B57, 64 (1973).

[44] J.A. Oller and E. Oset, Nucl. Phys. A629, 739 (1998).

[45] F. Guerrero and J. A. Oller (in preparation).

[46] E. Oset and A. Ramos, Nucl. Phys. A635, 99 (1998).

[47] P. Schuck, W. Norenberg, and G. Chanfray, Z. Phys. A 330, 119 (1988); H.C. Chiang, E. Oset, and M.J. Vicente-Vacas, Nucl. Phys. A (to be published), nucl-th/9712047.

[48] A. Dobado, M.J. Herrero, and T.N. Truong, Phys. Lett. B 235, 129 (1990); A. Dobado, M.J. Herrero, and J. Terrón, Z. Phys. C 50, 205 (1991); 50, 465 (1991); J.R. Peláez, Phys. Rev. D 55, 4193 (1997). 